\title{
Effects of hospital-wide interventions to improve care for frail older inpatients: a systematic review
}

\section{Order of authors}

Franka C Bakker, MSc ${ }^{1}$ Sarah HM Robben, $\mathrm{MD}^{1}$ and Marcel GM OldeRikkert, MD, $\mathrm{PhD}^{1}$

${ }^{1}$ Radboud University Nijmegen Medical Centre, Department of Geriatrics, Nijmegen, the Netherlands

Email addresses of (co-)authors:

Franka C. Bakker, MSc: f.bakker@ger.umcn.nl

Sarah H.M. Robben, MD: s.robben@ger.umcn.nl

Marcel G.M. Olde Rikkert, MD, PhD: m.olde-rikkert@ger.umcn.nl

Key words: hospitals; frail elderly; intervention; quality of care; systematic review 


\begin{abstract}
Background: Although it is widely recognized that frail older persons need adaptation of healthcare services, it is unclear how hospital care in general can best be tailored to their frailty.
\end{abstract}

Objective: To systematically review the evidence for hospital-wide interventions for older patients.

Methods: Pubmed, Cochrane CENTRAL, Cinahl, and reference lists of included articles (1980-2009) were searched. Papers describing 1) randomized controlled trials, controlled clinical trials, controlled before-after studies or interrupted time-series, 2) patients $\geq 65$ years admitted to hospital, 3) hospital-wide organizational interventions, and 4) patient-related outcomes, quality of care, patient safety, resource use, or costs were included. Two reviewers extracted data and assessed risk of bias independently, according to Cochrane Effective Practice and Organization of Care Review Group guidelines.

Results: We included 20 articles out of 1175 . Mean age of study populations ranged from 74.2 to 85.8 years. Interventions included multidisciplinary (consultative) teams, nursing care models, structural changes in physical environment and/or changes in site of service delivery. Small or no effects were found on patient-related outcomes such as functional performance, length of stay, discharge destination, resource use and costs compared with usual care. Methodological quality evaluation showed data incompleteness and contamination as main sources of bias.

Conclusions: No single best hospital-wide intervention could be identified using strict methodological criteria. However, several interventions had positive results, and may be used in hospital practice. Since strict methodological designs are not optimal for evaluating highly complex interventions and settings, we recommend studying hospital-wide interventions for older persons using adapted quality and research criteria. 


\section{INTRODUCTION}

The quickly growing number of frail older surgical and non-surgical inpatients emphasizes the need to develop hospital-wide interventions to improve outcomes of hospital care. ${ }^{1}$ Hospital-wide interventions are system interventions, not restricted to medical specialties or departments, that are available for all older hospitalized patients. Comprehensive geriatric assessment (CGA) has been introduced and further developed to maintain or improve functioning in frail older patients, and has been proven to be effective when implemented ward-based (as opposed to inpatient geriatric consultation service). ${ }^{2-4} \mathrm{CGA}$ is a multidimensional, interdisciplinary diagnostic instrument designed to determine the medical, psychosocial and functional capabilities and limitations of elderly patients in order to develop a coordinated and integrated plan for treatment and long-term follow-up. ${ }^{3}$ However, having only one geriatric ward cannot improve care for all frail hospitalized older patients, since persons older than 65 years currently form the largest proportion of all inpatients. In addition, despite the development of CGA, there is still a high risk of poor functional outcomes and dependency during ${ }^{5}$ or after $^{6}$ hospitalization. Delirium and falls are examples of major and often preventable adverse events ${ }^{7,8}$, which quickly increase with age. ${ }^{9}$

Thus, enforced by healthcare reforms, interest in effective and efficient care models for older patients, next to existing geriatric specialized wards, is increasing. ${ }^{10}$ Therefore, the primary objective of this article is to systematically review the evidence for hospital-wide interventions for frail older patients. 


\section{METHODS}

\section{Data Sources}

We performed a search of Pubmed, Cochrane CENTRAL, and Cinahl, from 1 January 1980 to 15 May 2009, including only articles written in English. For Pubmed a comprehensive search strategy was developed (Appendix A), which was adapted for the other databases (Appendices B and C). Methodological search filters for Medline (for Pubmed) and Cinahl were used as described by the Cochrane Effective Practice and Organization of Care Group (EPOC).(www.epoc.cochrane.org) The snowball method was used to manually identify relevant references from the reference lists of included articles.

\section{Study Selection}

We explicitly searched for interventions that were developed to be implementable on a hospital-wide basis and therefore available for all hospitalized older patients. We defined hospital-wide interventions as integrated practices throughout the hospital system of care delivery for older patients, which are not restricted to medical departments or-specialties (e.g. geriatric departments as the only place providing special attention to older patients and therefore available only for the, clearly visible, frailest patients). The term 'frailty' was primarily used as a term to retrieve studies of interest, but not as an in- or exclusion criterion, since there is still much debate on its definition. Studies were considered for inclusion when they: 1) included patients 65 years or older and acutely admitted to hospital, 2) described an organizational intervention designed and piloted or implemented to improve hospital-wide quality, safety or effectiveness of care for (frail) inpatients $\geq 65$ years, 3 ) reported outcomes related to either quality of care, patient safety, patient-related outcomes, resource use or costs, and 4) were a randomized controlled trial (RCT), controlled clinical trial (CCT), controlled before-after study (CBA) or interrupted time-series (ITT). Studies describing 1) medical 
specialty-, disease- or disability-specific interventions, 2) pre- or post-hospital interventions (e.g. improvement of transfers), 3) specialized hospitals (e.g. rehabilitation, long-term, intermediate care), or 4) single-component interventions (e.g. use of falls prevention protocol) were excluded. The first and fourth exclusion criteria were chosen as we are looking for interventions which serve, in concordance to CGA, all frail older patients with their complex and heterogeneous health problems.

\section{Data Extraction \& Quality Assessment}

Two researchers (FB and SR) conducted the initial search by independently examining each title and available abstract. Retrieved full-text studies were independently reassessed (FB and SR). A third researcher (MOR) was consulted in case of disagreement. Data were collected based on the checklist of the Cochrane EPOC Review Group, and abstracted using a modified version of the EPOC data extraction form (Appendix D).(www.epoc.cochrane.org) Data collected included details of the intervention, patients and providers, setting, and primary outcomes. Quality assessment was included by using the most recent 2009 EPOC form, which includes nine standard criteria to assess the risk of bias: randomization, allocation concealment, baseline comparability, incomplete outcome data, blinding of participants, providers or outcome assessors, selective outcome reporting, or other risks of bias. A consensus-based risk of bias table was constructed.

\section{Data Synthesis \& Analysis}

Conducting a meta-analysis was not feasible. Results of included studies were therefore analyzed by making qualitative, descriptive summaries. We show results as presented by original studies. Additionally, effect sizes (Cohen's $d$ ) were calculated (d of 0.20 judged as 
small, 0.50 as medium, and 0.80 as large), when standard deviations and means were presented in the original article (further details: Appendix F). 


\section{RESULTS}

\section{Included Studies}

The search strategy identified 1175 citations of which 11 articles could be included for analysis. The snowball method yielded an additional 9 articles. Figure 1 details the results of the steps in the search strategy. The 20 included articles represent results of 17 studies (12 RCT's and 5 CCT's).

\section{Study Characteristics}

Characteristics of included studies are shown in Table 1. More detailed information is available in Appendix Table 1. The mean age of the population varied from 74.2 to 85.8 years across studies. Whereas most studies used age to select a frail population or selected frail patients during the intervention, seven of the included studies used additional criteria to select frail inpatients. ${ }^{11-18}$ As for the location of the intervention, one study described an intervention starting in the Emergency Department ${ }^{17}$, whereas the other studies describe interventions initiated at general medical wards. All but two studies set up multidisciplinary teams; these two studies only made structural changes in physical environment and/or site of service delivery. ${ }^{14,17}$ Four studies initiated, in addition to a multidisciplinary team, an intervention including modifications of the physical environment. ${ }^{14,19-21}$ In seven studies the main providers of the intervention were nurses. ${ }^{14,17,19-23}$ In one study the main providers of care were rehabilitation staff. ${ }^{24}$ In the other studies (geriatric) physicians were the responsible professionals and/or main providers of the intervention. Interventions (I) were compared to controlled usual care (C) as provided throughout the hospital, prior or next to the interventions.

\section{Risk of bias}


On average, we found two main sources of potential bias (Table 2). For 14 articles, it was unclear whether or not the incomplete outcome data had been addressed adequately (i.e. it was not specified whether missing outcome measures potentially biased the results as presented in the article). Contamination was inadequately addressed or not described in 19 articles.

\section{Effectiveness of Interventions}

Primary outcomes were functional performance, length of stay, mortality, discharge destination, readmission, complications, resource use and costs (Table 1; further details: Appendix Table 2).

\section{Functional outcomes}

Fourteen studies presented results on functional patient outcomes. Of these, five studies (four Geriatric Consultation Teams $(\mathrm{GCT})^{11,13,15,22}$, one dayroom ${ }^{14}$ ) showed significant effects for patients in the intervention group on mental health, emotional or cognitive status. Three studies (two GCT ${ }^{12,16}$, one Acute Care for Elders unit ${ }^{20}$ ) demonstrated significant improvements in physical outcomes.

\section{Mortality}

Of five studies having mortality as one of the primary outcome measures, two $\left(\mathrm{GCT}^{12,25}\right)$ revealed positive significant results on survival or mortality at 6 months follow-up.

\section{Length of Stay}

Of nine studies studying length of stay (LOS), one (primary nursing model of care ${ }^{19}$ ) had a significant shorter LOS in one of the two experimental sites. 


\section{Discharge Destination}

Eight studies focused on discharge destination. Of these, one (primary nursing model of care $^{19}$ ) showed a statistically significantly higher nursing homes admission rate and one $\left(\mathrm{GCT}^{18}\right)$ had a significant lower number of nursing home admissions at 12 months.

\section{Resource Use}

Two studies studied in-hospital resource use, of which one $\left(\mathrm{GCT}^{11}\right)$ showed a significant higher rate of referral to rehabilitation services. Six studies measured post-discharge resource use. Three (two GCT ${ }^{18,11}$, one geriatric-based ward ${ }^{26}$ ) showed a significant lower average number of nursing home days per patients at 12 months, higher mean number of referrals to community services, or a higher number of outpatients visits per patients to a physical or occupational therapist up to three months follow-up.

\section{Readmission}

$\mathrm{One}^{25}$ of five studies (four $\mathrm{GCT}^{12,13,25-27}$, one geriatric-based ward ${ }^{25}$ ) presenting data on rehospitalization showed fewer readmissions per patient, at 6 months follow-up.

\section{Complications}

A primary nursing model of care and a GCT registering hospital-acquired complications showed no statistically significant results. ${ }^{19,28}$

\section{Economic Variables}

Four studies evaluated costs of the intervention. ${ }^{18,19,22,26}$ Two (primary nursing model of care $^{19}, \mathrm{GCT}^{18}$ ) demonstrated lower costs. 


\section{DISCUSSION}

This systematic review assessing the effects of interventions to improve hospital-wide care for older inpatients showed that no single best evidence-based practice can be described, that improves quality of care, safety and effectiveness. Different forms of geriatric consultation teams were partly effective in improving patient-related outcomes and process quality measures. Additionally, nursing models of care, wards admitting all older patients and environmental adaptations were found, with heterogeneous effects in different settings. The designs are methodologically not sufficiently strict and the studies too heterogeneously described to allow summary statistics or a Cochrane high-quality evidence rating.

The heterogeneity in the studies can be explained in several ways. First, hospitals differ from site to site in catchment area and associated demographic and sociocultural setting, referral practice, specialization, staff, and overall quality and safety of care. Consequently, care interventions highly differ, even if they are based on a similar model of care. ${ }^{19}$ In addition, positive effects across studies were found on different outcomes and positive outcomes only showed moderate or small effects (effect sizes ranged from .16 to .37). Ten studies introduced an intervention including GCT's principles, of which four studies demonstrated no significant effects on their primary outcomes. Three showed small effects in mental status or mood. ${ }^{11,13,22}$ One of these ten showed positive effects on the Barthel score and survival ${ }^{12}$, and one in survival and readmission rates. ${ }^{25}$ Four studies which introduced an intervention with nurses as main providers, found no or small effects, which they ascribed to limited availability of resources and thus limited intensity of the intervention. Of the two ACE unit studies which intended to be implemented hospital-wide eventually, one had positive results on functional outcomes. ${ }^{20}$ The other explains improvements in usual care as the main cause for the lack of significant results. ${ }^{21}$ It is also possible that usual care was contaminated 
by the intervention in the majority of studies, which may have influenced the ability to show positive effects.

However, although effects are small, positive results are definitely important in such a frail population. About 22 percent of persons older than 80 years who are admitted to a hospital die within one year after discharge ${ }^{29}$, and the average time for partial or full recovery after hospitalization is 18 months ${ }^{30}$. Therefore, each step forward is important in effectiveness of hospital care, such as stabilization of functional performance, and is an important positive result. Studies showing no significant improvement of overall functional status, mortality or readmissions, but which do show a tendency towards less functional decline ${ }^{11,13,15,21,25,31}$, mortality $^{24}$, or readmission ${ }^{12,26}$ are therefore very valuable.

\section{Comparison with published literature}

As far as we know we are the first to review hospital-wide interventions, though there are articles describing intervention studies included here. Landefeld et al. summarized lessons to be learned from Geriatric Evaluation and Management (GEM) departments, ACE units, and the HELP set-up. ${ }^{32}$ Similarly, Palmisano-Mills identified the implementation of different versions of four models of integrated care for older patients (including HELP, ACE units, NICHE, and a Model of Transitional Care) in 24 hospitals in Connecticut. She found that few hospitals have implemented the original models, but that the majority successfully implemented key components of the care models as well as their own innovative protocols. ${ }^{33}$ However, the success of these implementation projects was never substantiated in an RCT.

This review only included RCT's and CCT's, which has led to exclusion of studies with lower methodological quality. However, as Table 2 shows, none of the included studies still is without serious risk of bias, only one study showing protection against contamination. This evokes the question whether these studies are methodologically flawed, but could have 
been performed better, or whether systematic review techniques applying strict methodological Cochrane criteria are less appropriate in selecting these complex evaluations of service delivery and organization of care. ${ }^{34}$ As such, Harari and colleagues evaluated a hospital-wide intervention in which an Older Persons' Assessment and Liaison (OPAL) team improved processes of care. Although the study design did not meet our inclusion criteria, results of this study are promising in terms of effectiveness and efficiency. ${ }^{35}$ The same conclusion may be drawn for the Older Adult Services Inpatient Strategies (OASIS) program, which aims for improvement of care for older patients throughout the hospital. ${ }^{36}$

Additionally, we only found one study on the hospital-wide Hospital Elder Life Program (HELP), which could be included in our review. ${ }^{23}$ The others were excluded due to the study design or e.g. a focus on delirium in a specific patient group. Not including such studies based on design criteria is debatable, as such studies seem to support the evidencebased practice of implementation of HELP and subsequently prevent cognitive and functional decline. ${ }^{37,}{ }^{38}$ (http://elderlife.med.yale.edu) This also applies for the Nurses Improving Care for Health System Elders (NICHE) program, which has evolved into a national USA/Canadian geriatric nursing program.(http://hartfordign.org) Our Cochrane review criteria yielded only two studies implementing a program based on NICHE. ${ }^{39,39,40}$ A third intervention of which we could only include two articles is the Acute Care for Elders Unit (ACE) ${ }^{20,21}$, which is mentioned as the state-of-the-art care model to improve hospital-wide care for older adults. Also other studies support the evidence that development of ACE units can improve health and functioning of older persons, without increasing health care costs. ${ }^{41,42}$

\section{Limitations}

It should be noted that our snowball method has favored older studies. However, recently a non RCT study of a proactive geriatrics consultation model was published ${ }^{43}$, indicating that 
hospitals are still using similar models of care to improve care for frail older patients. The same accounts for the ACE unit, which was developed in the early 1990s, where efforts are still made to get (adapted versions of) this model of care disseminated throughout hospitals. ${ }^{44}$

\section{Future directions}

The key message for hospital practice is that one should investigate what works best in a specific hospital, preferably by piloting an intervention that uses effective and innovative intervention components, and incorporates the barriers and facilitators of implementation as well (Appendix Table 3). This stepwise procedure is proposed by the Medical Research Council's framework for complex interventions. ${ }^{45}$ Dynamic and complex healthcare organizations, such as modern hospitals, require innovative interventions as well as innovative research designs that are flexible enough to allow changes to be made during the intervention (e.g. time series analyses, before-after studies). ${ }^{46}$ For innovative hospital reform interventions, this can be realized by transition management, which adapt interventions with regard to the facilitators and barriers met during the implementation process. For evaluation, apart from more flexible options than RCTs, we suggest to use quality indicators (QI's) to monitor effects on the major health problems that are targeted. For example, the Assessing Care of Vulnerable Elders (ACOVE) indicators are objective and comprehensive measures, which are a useful starting point for developing site-specific QI's. ${ }^{47,48}$ In addition, to be able to compare outcomes in older patients within and between studies, methods for incorporating key descriptors like cognitive and physical functioning to adjust for different case-mixes should be introduced into routine clinical practice. ${ }^{49}$ Another innovative and promising evaluation of health care reform by complex interventions is to follow the framework that has recently been proposed by Porter et al. ${ }^{50}$ This framework defines value measures as outcomes in evaluating healthcare practices. Porter provides a framework through which this value (or: 
performance) of an intervention can be identified, using multilevel patient-oriented outcomes related to their full costs. For both scientific and societal evaluation, it would be an important step forward to be able to continuously monitor the value of an intervention for a specific inpatient group like frail older patients.

\section{Conclusion}

The current aging of the population and developments in hospital care explicitly call for comprehensive interventions aimed to improve care for all frail older patients throughout the hospital. While implementing evidence-based practices is stimulated, only a few hospitalwide intervention RCT studies could be identified. It is urgently needed to study alternative approaches and to set adjusted scientific standards to gain firm evidence-based improvements in hospital-wide care for frail older patients. 


\section{ACKNOWLEDGEMENTS}

We thank Maurits van Tulder, $\mathrm{PhD}$, and Marieke Perry, MD, for their support and comments on the review.

Contributors: All authors contributed to the study design, selection of articles, data extraction, analysis and interpretation, and writing of the manuscript.

Competing interests: none declared

Funding: The work was made possible by grant 60-6190-098-272 and grant 60-61900-98129 of the National Programme for Elderly Care, coordinated and sponsored by ZonMw, The Netherlands, organization for health research and development. The funding source did not participate in the literature search, data analysis, or interpretation of results. 


\begin{tabular}{|c|c|c|c|c|c|c|}
\hline STUDY & DESIGN & PATIENTS & INTERVENTION & CONTROL & PRIMARY OUTCOMES & RESULTS \\
\hline $\begin{array}{l}\text { Campion }^{27} \text {, } \\
1983 \\
\text { general teaching } \\
\text { hospital, } \\
\text { USA }\end{array}$ & $\begin{array}{l}\text { CCT, } \\
\text { pilot study } \\
\text { Median } \\
\text { follow-up } \\
10.5 \\
\text { months }\end{array}$ & $\begin{array}{l}\text { All patients } \geq 75 \\
\text { years admitted to } \\
\text { teaching medical } \\
\text { wards } \\
I / C^{\ddagger}: 46 / 86\end{array}$ & $\begin{array}{l}\mathrm{GCT}^{\S}(a / b / c / d / e / f / g / h / i) \text { provided CGA, } \\
\text { consultation and in-patient follow-up to all } \\
\text { admitted patients, with the attending physician } \\
\text { being responsible for implementation of written } \\
\text { or verbally communicated recommendations. }\end{array}$ & $\begin{array}{l}\text { Two similar } \\
\text { wards, } \\
\text { GCT not } \\
\text { available }\end{array}$ & $\begin{array}{l}\text { Rehospitalization } \\
\text { LOS } \\
\text { Discharge destination } \\
\text { Resource use in-hospital }\end{array}$ & $\begin{array}{l}\mathrm{u} \\
\mathrm{u} \\
\mathrm{u} \\
\mathrm{u}\end{array}$ \\
\hline $\begin{array}{l}\text { Collard }^{19} \\
1985 \\
2 \text { community } \\
\text { hospitals, } \\
\text { USA }\end{array}$ & $\begin{array}{l}\text { RCT } \\
\text { Follow-up } \\
6 \text { months }\end{array}$ & $\begin{array}{l}\text { All medical/surgical } \\
\text { patients } \geq 65 \text { years } \\
\text { I/C: } 218 / 477\end{array}$ & $\begin{array}{l}10 \text { bed Geriatric Special Care Unit in an } \\
\text { existing space adopting a primary nursing } \\
\text { model of care available for all randomly } \\
\text { admitted patients. }\end{array}$ & $\begin{array}{l}\text { Task-oriented } \\
\text { model of care } \\
(c / k)\end{array}$ & $\begin{array}{l}\text { Complications } \\
\text { LOS } \\
\text { Discharge destination } \\
\text { Use of restraints } \\
\text { Costs }\end{array}$ & $\begin{array}{c}\mathrm{ns} / \mathrm{NS} \\
\mathrm{ns} /+ \\
\mathrm{ns} /+ \\
\mathrm{ns} / \mathrm{ns} \\
\mathrm{ns} /+\end{array}$ \\
\hline $\begin{array}{l}\text { Becker }^{28 \|} \\
1987 \\
\text { VA Medical } \\
\text { Center } \\
\text { USA }\end{array}$ & $\begin{array}{l}\mathrm{RCT} \\
\text { No follow- } \\
\text { up }\end{array}$ & $\begin{array}{l}\text { All patients } \geq 75 \\
\text { years admitted to } \\
\text { medical, psychiatric } \\
\text { and } \\
\text { surgical wards } \\
\text { I/C: } 92 / 89\end{array}$ & $\begin{array}{l}\text { GCT }(a / b / c / l) \text { placed specific prioritized list of } \\
\text { recommendations in charts, discussed it directly } \\
\text { with ward staff, and provided in-hospital } \\
\text { follow-up for all admitted patients. }\end{array}$ & $\begin{array}{l}\text { GCT placed } \\
\text { only problem } \\
\text { list in charts }\end{array}$ & $\begin{array}{l}\text { Hospital-acquired } \\
\text { complications }\end{array}$ & NS \\
\hline $\begin{array}{l}\text { Saltz }^{51 \|}, \\
1988\end{array}$ & $\begin{array}{l}\text { Follow-up } \\
6 \text { months }\end{array}$ & “ & “ & & Discharge destination & NS \\
\hline $\begin{array}{l}\text { McVey }^{31 \|}, \\
1989\end{array}$ & $\begin{array}{l}\text { No follow- } \\
\text { up }\end{array}$ & $I / C: 88 / 90$ & “ & & Functional outcomes & NS \\
\hline
\end{tabular}


Table 1. Characteristics and results on primary outcomes in the 20 studies included

\begin{tabular}{|c|c|c|c|c|c|c|}
\hline STUDY & DESIGN & PATIENTS & INTERVENTION & CONTROL & PRIMARY OUTCOMES* & $\underset{\dagger}{\text { RESULTS }}$ \\
\hline $\begin{array}{l}\text { Gayton }^{24}, \\
1987 \\
\text { teaching hospital, } \\
\text { Canada }\end{array}$ & $\begin{array}{l}\text { CCT } \\
\text { Follow-up } \\
6 \text { months }\end{array}$ & $\begin{array}{l}\text { All patients } \geq 70 \\
\text { years directly } \\
\text { admitted to } \quad(4) \\
\text { general medical } \\
\text { wards from ED } \\
\text { I/C: } 222 / 182\end{array}$ & $\begin{array}{l}\text { GCT }(a / b / c / d / e) \text { provided consultation, } \\
\text { suggestions and in-hospital follow-up to } \\
\text { randomly assigned patients after informal } \\
\text { contacts with ward staff and weekly ward } \\
\text { rounds. }\end{array}$ & Usual care & $\begin{array}{l}\text { Functional outcomes } \\
\text { LOS } \\
\text { Discharge destination } \\
\text { Resource use post-discharge }\end{array}$ & $\begin{array}{l}\mathrm{u} \\
\mathrm{u} \\
\mathrm{u} \\
\mathrm{u}\end{array}$ \\
\hline
\end{tabular}


Table 1. Characteristics and results on primary outcomes in the 20 studies included

\begin{tabular}{|c|c|c|c|c|c|c|}
\hline STUDY & DESIGN & PATIENTS & INTERVENTION & CONTROL & PRIMARY OUTCOMES & RESULTS \\
\hline $\begin{array}{l}\text { Fretwell }^{22}, \\
1990 \\
\text { general hospital, } \\
\text { USA }\end{array}$ & $\begin{array}{l}\text { RCT } \\
\text { Follow-up } \\
6 \text { months }\end{array}$ & $\begin{array}{l}\text { All patients } \geq 75 \\
\text { years } \\
\text { I/C: } 221 / 215\end{array}$ & $\begin{array}{l}18 \text { bed Senior Care Unit where a GCT } \\
(\mathrm{a} / \mathrm{b} / \mathrm{c} / \mathrm{d} / \mathrm{h} / \mathrm{m}) \quad \text { provided assessment, } \\
\text { recommendations in chart, in-hospital and post- } \\
\text { discharge follow-up to all randomized patients, } \\
\text { with a focus on functional assessment by nurses } \\
\text { within routine admission evaluations, and the } \\
\text { attending physician being the main responsible. }\end{array}$ & $\begin{array}{l}\text { Usual care at } \\
\text { traditional } \\
\text { medical and } \\
\text { surgical wards } \\
\text { (consult } \\
\text { geriatrician } \\
\text { possible) }\end{array}$ & $\begin{array}{l}\text { Functional outcomes } \\
\text { - } \quad \text { functional; ADL } \\
\text { - } \quad \text { mental; MMSE } \\
\text { - emotional; SDS } \\
\text { LOS } \\
\text { Costs }\end{array}$ & $\begin{array}{l}\mathrm{ns} \\
\mathrm{ns} \\
+ \\
\mathrm{ns} \\
\mathrm{ns}\end{array}$ \\
\hline $\begin{array}{l}\text { Hogan }^{12}, \\
1990 \\
\text { general hospital, } \\
\text { Canada }\end{array}$ & $\begin{array}{l}\text { CCT } \\
\text { Follow-up } \\
12 \text { months }\end{array}$ & $\begin{array}{l}\text { All patients } \geq 75 \\
\text { years admitted to the } \\
\text { Department of } \\
\text { Medicine on an } \\
\text { emergency basis, } \\
\text { with Geriatric Status } \\
\text { categories } 3,4,5 \\
I / C: 66 / 66\end{array}$ & $\begin{array}{l}\text { GCT }(a / b / c / d / e / h / n) \text { provided assessment, in- } \\
\text { hospital follow-up and post-hospital follow-up } \\
\text { for all patients through initial contact by } \\
\text { physician-to-physician consultation, } \\
\text { involvement of other members as required. }\end{array}$ & $\begin{array}{l}\text { Usual care } \\
\text { (geriatric } \\
\text { services } \\
\text { available in } \\
\text { hospital) }\end{array}$ & $\begin{array}{l}\text { Functional outcomes } \\
\text { - } \quad 3 \text { months } \\
\text { - } \quad 6 \text { months } \\
\text { - } \quad 12 \text { months } \\
\text { Discharge destination } \\
\text { Mortality } \\
\text { - } \quad \text { in-hospital } \\
\text { - } \quad 6 \text { months } \\
\text { - } \quad 12 \text { months } \\
\text { Rehospitalization }\end{array}$ & $\begin{array}{l}\mathrm{ns} \\
+ \\
\mathrm{ns} \\
\mathrm{ns}\end{array}$ \\
\hline $\begin{array}{l}\text { Inouye }^{23}, \\
1993 \\
\text { teaching hospital, } \\
\text { USA }\end{array}$ & $\begin{array}{l}\text { CCT } \\
\text { No follow- } \\
\text { up }\end{array}$ & $\begin{array}{l}\text { All patients } \geq 70 \\
\text { years admitted to } \\
\text { one of } 5 \text { general } \\
\text { medical units } \\
\text { I/C: }(42+43) / 131\end{array}$ & $\begin{array}{l}\text { Introducing the Yale Geriatric Care Program, a } \\
\text { nursing-centered model of care at } 2 \text { acute } \\
\text { medical units ( } 1 \text { nurse-only, } 1 \text { geriatrician- } \\
\text { nurse), where a care team }(a / b) \text { screened all } \\
\text { patients for frailty, provided in-hospital follow- } \\
\text { up for all frail patients, and educated all nurses. }\end{array}$ & $\begin{array}{l}\text { Usual care, } \\
3 \text { medical units }\end{array}$ & Functional outcomes & ns \\
\hline
\end{tabular}


Table 1. Characteristics and results on primary outcomes in the 20 studies included

\begin{tabular}{|c|c|c|c|c|c|c|}
\hline STUDY & DESIGN & PATIENTS & INTERVENTION & CONTROL & PRIMARY OUTCOMES* & $\underset{\dagger}{\text { RESULTS }}$ \\
\hline $\begin{array}{l}\text { Thomas }^{25} \\
1993 \\
\text { community } \\
\text { hospital, } \\
\text { USA }\end{array}$ & $\begin{array}{l}\mathrm{RCT} \\
\text { Follow-up } \\
6 \text { months }\end{array}$ & $\begin{array}{l}\text { All patients } \geq 70 \\
\text { years } \\
\text { I/C: } 62 / 58\end{array}$ & $\begin{array}{l}\text { GCT }(b / c / d / h / j / m / o) \text { provided assessment, } \\
\text { recommendations in charts with copies to } \\
\text { attending physician's office, and in-hospital } \\
\text { follow-up for all inpatients. }\end{array}$ & $\begin{array}{l}\text { No } \\
\text { recommend- } \\
\text { dations, no } \\
\text { subsequent } \\
\text { visits }\end{array}$ & $\begin{array}{l}\text { Functional outcomes } \\
\text { Rehospitalization } \\
\text { Mortality } \\
\text { - } \quad 6 \text { months } \\
\text { - } \quad 12 \text { months } \\
\text { LOS } \\
\text { Discharge destination } \\
\text { Resource use post-discharge } \\
\text { - } \quad \text { community services } \\
\text { - } \quad \text { outpatient visits }\end{array}$ & $\begin{array}{c}\text { NS } \\
+ \\
+ \\
+ \\
\text { NS } \\
\text { NS } \\
\text { u } \\
\\
\text { NS } \\
\text { NS }\end{array}$ \\
\hline $\begin{array}{l}\text { Winograd }^{13}, \\
1993 \\
\text { VA Medical } \\
\text { Center, } \\
\text { tertiary care } \\
\text { teaching hospital, } \\
\text { USA }\end{array}$ & $\begin{array}{l}\mathrm{RCT} \\
\text { Follow-up } \\
12 \text { months }\end{array}$ & $\begin{array}{l}\text { All male patients } \\
\geq 65 \text { years admitted } \\
\text { to acute medical and } \\
\text { surgical wards, and } \\
\text { functionally } \\
\text { impaired with one of } \\
\text { proxy criteria for } \\
\text { frailty } \\
\text { I/C: } 99 / 98\end{array}$ & $\begin{array}{l}\text { GCT }(a / b / c / l / p) \text { provided assessment and in- } \\
\text { hospital follow-up for all inpatients screened as } \\
\text { frail, placed recommendations in charts, } \\
\text { discussed them with the primary care team, and } \\
\text { provided in-service education when needed. }\end{array}$ & $\begin{array}{l}\text { Usual care, } \\
\text { not evaluated } \\
\text { by GCT }\end{array}$ & $\begin{array}{l}\text { Functional outcomes } \\
\text { - } \quad \text { IADL } \\
\text { - } \quad \text { MMSE } \\
\text { - } \quad \text { PSMS } \\
\text { Mortality } \\
\text { LOS } \\
\text { Discharge destination } \\
\text { Resource use post-discharge }\end{array}$ & $\begin{array}{c}\text { NS } \\
+ \\
\text { NS } \\
\text { NS } \\
\text { NS } \\
\text { NS } \\
\text { NS }\end{array}$ \\
\hline
\end{tabular}


Table 1. Characteristics and results on primary outcomes in the 20 studies included

\begin{tabular}{|c|c|c|c|c|c|c|}
\hline STUDY & DESIGN & PATIENTS & INTERVENTION & CONTROL & PRIMARY OUTCOMES & $\underset{\dagger}{\text { RESULTS }}$ \\
\hline $\begin{array}{l}\text { Clark }^{14}, \\
1995 \\
\text { teaching hospital, } \\
\text { USA }\end{array}$ & $\begin{array}{l}\text { CCT } \\
\text { No follow- } \\
\text { up }\end{array}$ & $\begin{array}{l}\text { All patients } \geq 65 \\
\text { years, at risk of falls, } \\
\text { mental status } \\
\text { changes or } \\
\text { associated diagnoses } \\
I / C: 40 / 40\end{array}$ & $\begin{array}{l}\text { Dayroom (hospital room with special features } \\
\text { and activities) on a medical nursing unit, staffed } \\
\text { by existing nurses, available for } 4 \text { selected } \\
\text { patients at a time. }\end{array}$ & $\begin{array}{l}\text { Usual care in } \\
\text { hospital rooms }\end{array}$ & $\begin{array}{l}\text { Functional outcomes } \\
\text { - ADL } \\
\text { - SPMSQ score } \\
\text { LOS } \\
\text { Resource use in-hospital }\end{array}$ & $\begin{array}{l}\mathrm{u} \\
- \\
\mathrm{u} \\
+\end{array}$ \\
\hline $\begin{array}{l}\text { Landefeld }^{20}, \\
1995 \\
\text { teaching hospital, } \\
\text { USA }\end{array}$ & $\begin{array}{l}\text { RCT } \\
\text { Follow-up } \\
3 \text { months }\end{array}$ & $\begin{array}{l}\text { All patients } \geq 70 \\
\text { years acutely } \\
\text { admitted for general } \\
\text { medical care } \\
I / C: 327 / 324\end{array}$ & $\begin{array}{l}14 \text { bed Acute Care for Elders unit, consisting of } \\
\text { a specially designed environment, patient- } \\
\text { centered care, discharge planning, and medical } \\
\text { review, with the primary nurse being the key } \\
\text { provider in providing care for all inpatients. }\end{array}$ & $\begin{array}{l}\text { Usual care in } \\
\text { another } \\
\text { general } \\
\text { medical ward }\end{array}$ & Functional outcomes & + \\
\hline $\begin{array}{l}\text { Reuben }^{15}, \\
1995 \\
\text { HMO: } 4 \text { medical } \\
\text { centers, } \\
\text { USA }\end{array}$ & $\begin{array}{l}\text { Multi-site } \\
\text { RCT } \\
\text { Follow-up } \\
12 \text { months }\end{array}$ & $\begin{array}{l}\text { All patients } \geq 65 \\
\text { years with } \geq 1 \text { of } 13 \\
\text { screening criteria } \\
\text { I/C: } 1337 / 1016\end{array}$ & $\begin{array}{l}\text { GCT }(a / b / c) \text { provided written recommendations } \\
\text { to attending physician and primary care } \\
\text { physician (with the geriatrician being able to } \\
\text { order small therapies directly), in-hospital } \\
\text { follow-up, and post-discharge follow-up to all } \\
\text { eligible patients. }\end{array}$ & Usual care & $\begin{array}{l}\text { Functional outcomes } \\
\text { - } \quad \text { ADL } \\
\text { - } \quad \text { Social activities } \\
\text { - } \quad \text { Mental health } \\
\circ 3 \text { months } \\
\circ 12 \text { months } \\
\text { - Health perceptions } \\
\circ 3 \text { months } \\
\circ 12 \text { months } \\
\text { Mortality }\end{array}$ & $\begin{array}{l}\mathrm{ns} \\
\mathrm{ns} \\
+ \\
\mathrm{ns}\end{array}$ \\
\hline
\end{tabular}


Table 1. Characteristics and results on primary outcomes in the 20 studies included

\begin{tabular}{|c|c|c|c|c|c|c|}
\hline STUDY & DESIGN & PATIENTS & INTERVENTION & CONTROL & PRIMARY OUTCOMES & $\underset{\dagger}{\text { RESULTS }}$ \\
\hline $\begin{array}{l}\text { Asplund } \\
2000 \\
\text { university } \\
\text { hospital, } \\
\text { Sweden }\end{array}$ & $\begin{array}{l}\text { RCT } \\
\text { Follow-up } \\
3 \text { months }\end{array}$ & $\begin{array}{l}\text { All patients } \geq 70 \\
\text { years admitted } \\
\text { acutely } \\
\text { from ED to general } \\
\text { medical wards } \\
\text { I/C: } 190 / 223\end{array}$ & $\begin{array}{l}11 \text { bed geriatrics-based ward, comprised of a } \\
\text { care team }(a / d / e / h / p) \text { who provide assessment, } \\
\text { early rehabilitation, and intense discharge } \\
\text { planning for all unselected randomized patients, } \\
\text { and an internist having main responsibility } \\
\text { during the acute phase. }\end{array}$ & $\begin{array}{l}\text { Usual care on } \\
2 \quad \text { existing } \\
\text { general } \\
\text { medical wards, } \\
\text { each } 30 \text { beds } \\
\text { (c) }\end{array}$ & $\begin{array}{l}\text { Functional outcomes } \\
\text { Rehospitalization } \\
\text { Costs } \\
\text { Resource use post-discharge } \\
\text { - } \quad \text { Physician } \\
\text { - } \quad \text { Nurse } \\
\text { - } \quad \text { Physio-/occupational }\end{array}$ & $\begin{array}{l}\mathrm{ns} \\
\mathrm{ns} \\
+\end{array}$ \\
\hline $\begin{array}{l}\text { Counsell }^{21} \\
2000 \\
\text { community } \\
\text { teaching hospital, } \\
\text { USA }\end{array}$ & $\begin{array}{l}\mathrm{RCT} \\
\text { Follow-up } \\
12 \text { months }\end{array}$ & $\begin{array}{l}\text { All patients } \geq 70 \\
\text { years } \quad \text { admitted } \\
\text { acutely to medicine } \\
\text { or family practice } \\
\text { I/C: } 767 / 764\end{array}$ & $\begin{array}{l}34 \text { beds Acute Care for Elders unit, consisting } \\
\text { of a specially designed environment, patient- } \\
\text { centered care, early discharge planning and } \\
\text { medical review, with nurses having a key role } \\
\text { in providing care for all inpatients. }\end{array}$ & $\begin{array}{l}\text { Usual care } \\
\text { (GCT already } \\
\text { available in } \\
\text { hospital) }\end{array}$ & Functional outcomes & NS \\
\hline $\begin{array}{l}\text { Cohen } \\
2002 \\
\text { VA: } 11 \text { medical } \\
\text { centers, } \\
\text { USA }\end{array}$ & $\begin{array}{l}\text { Multi-site } \\
\text { RCT } \\
\text { Follow-up } \\
12 \text { months }\end{array}$ & $\begin{array}{l}\text { All frail patients } \geq 65 \\
\text { years admitted to } \\
\text { medical or surgical } \\
\text { wards } \\
I / C: 348 / 348\end{array}$ & $\begin{array}{l}\text { Geriatric Evaluation and Management } \\
\text { including a team }(a / b / c) \text { who provided } \\
\text { assessment, a care plan, and coordinated } \\
\text { preventive and management services for all } \\
\text { eligible patients. }\end{array}$ & Usual care & $\begin{array}{l}\text { Mortality } \\
\text { Health-related QoL } \\
\text { - } \quad \text { physical functioning } \\
\text { - } \quad \text { physical limitations } \\
\text { - } \quad \text { emotional limitations } \\
\text { - } \quad \text { bodily pain } \\
\text { - } \quad \text { energy } \\
\text { - } \quad \text { mental health } \\
\text { - } \quad \text { social activity } \\
\text { - } \quad \text { general health }\end{array}$ & $\begin{array}{c}\text { ns } \\
\text { NS } \\
\text { NS } \\
\text { NS } \\
+ \\
\text { NS } \\
\text { NS } \\
\text { NS } \\
\text { NS }\end{array}$ \\
\hline
\end{tabular}


Table 1. Characteristics and results on primary outcomes in the 20 studies included

\begin{tabular}{|c|c|c|c|c|c|c|}
\hline STUDY & DESIGN & PATIENTS & INTERVENTION & CONTROL & PRIMARY OUTCOMES & $\underset{\dagger}{\text { RESULTS }}$ \\
\hline $\begin{array}{l}\text { Phibbs }^{18 \|} \text {, } \\
2006\end{array}$ & & & & & $\begin{array}{l}\text { Resource use post-discharge } \\
\text { Costs } \\
\text { - } \quad \text { index hospitalization } \\
\text { - } \quad \text { after discharge }\end{array}$ & $\begin{array}{l}+ \\
+ \\
+\end{array}$ \\
\hline $\begin{array}{l}\text { Basic }^{17}, \\
2005 \\
\text { tertiary referral } \\
\text { hospital, } \\
\text { Australia }\end{array}$ & $\begin{array}{l}\mathrm{RCT} \\
\text { No follow- } \\
\text { up }\end{array}$ & $\begin{array}{l}\text { All older patients } \\
(78.7 \pm 6.4 \text { years) } \\
\text { presenting to the ED } \\
\text { with } \geq 1 \quad \text { screening } \\
\text { criteria } \\
\text { I/C: } 114 / 110\end{array}$ & $\begin{array}{l}\text { Aged Care Nurse in the Emergency Department } \\
\text { assisted in care of eligible patients by early } \\
\text { assessment, referral of patients and placing } \\
\text { recommendations in the medical file. }\end{array}$ & $\begin{array}{l}\text { Usual process } \\
\text { (geriatric } \\
\text { specialty } \\
\text { present in } \\
\text { hospital) }\end{array}$ & $\begin{array}{l}\text { Functional outcomes } \\
\text { Hospital admission } \\
\text { LOS }\end{array}$ & $\begin{array}{l}\mathrm{ns} \\
\mathrm{ns} \\
\mathrm{ns}\end{array}$ \\
\hline
\end{tabular}

Abbreviations: VA = Veteran Affairs; HMO = Health Maintenance Organization; LOS = length of stay; ADL = Activities of Daily Living; MMSE = Mini-Mental State Examination; SDS=Self

Rating Depression Scale; IADL= Instrumental Activities of Daily Living; PSMS = Physical Self-Maintenance Scale; SPMSQ = Short Portable Mental Status Questionnaire Score; (HR)QoL=

(Health-Related) Quality of Life; CGA = Comprehensive Geriatric Assessment

*Outcomes are the primary outcomes as described in the article.

${ }^{\dagger}$ Results: $+=$ statistically significant in favor of the experimental group with $\mathrm{p}<.05 ;-=$ statistically significant in favor of the control group with $\mathrm{p}<.05 ; \mathrm{NS}=$ not significant; ns $=$ described as not significant, but no p-value given; $\mathrm{u}=$ unknown/no statistical analyses performed. More detailed information is presented in Appendix table 2.

$\ddagger_{\mathrm{I}} / \mathrm{C}=$ Intervention Group/Control Group

${ }^{\S} \mathrm{GCT}=$ Geriatric Consultation Team; the disciplines composing the GCT are noted as: $\mathrm{a}=$ geriatrician, $\mathrm{b}=$ (geriatric) nurse (consultant/specialist/coordinator/practitioner/discharge planning), $\mathrm{c}$ $=$ social worker, $\mathrm{d}=$ physical therapist, $\mathrm{e}=$ occupational therapist, $\mathrm{f}=$ speech therapist, $\mathrm{g}=$ recreational therapist, $\mathrm{h}=$ dietitian, $\mathrm{i}=$ geropsychiatrist, $\mathrm{j}=$ physician, $\mathrm{k}=$ medical director, $\mathrm{l}=$ fellow in geriatrics, $\mathrm{m}=$ (clinical) pharmacist, $\mathrm{n}=$ pastoral carer, $\mathrm{o}=$ home health nurse, $\mathrm{p}=$ internist/internal medicine house officer

in same study

"same study 


\section{Table 2. Summary assessment of potential sources of bias}

\begin{tabular}{|c|c|c|c|c|c|c|c|c|c|}
\hline & 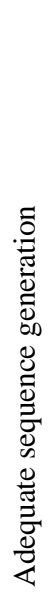 & 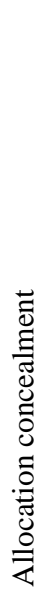 & 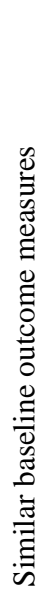 & 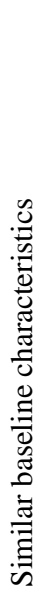 & 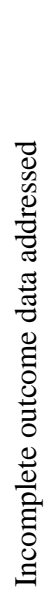 & 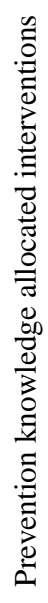 & 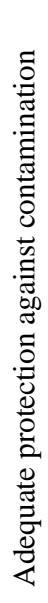 & 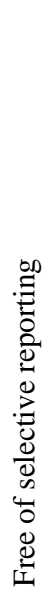 & 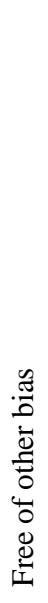 \\
\hline Campion, 1983, CCT & - & - & - & + & $?$ & $?$ & - & $?$ & - \\
\hline Collard, 1985, RCT & $?$ & $?$ & + & - & - & $?$ & - & - & - \\
\hline Becker $^{*}, 1987, \mathrm{RCT}$ & + & + & + & + & $?$ & + & - & + & - \\
\hline Saltz ${ }^{*}, 1988, \mathrm{RCT}$ & + & + & $?$ & $?$ & $?$ & + & - & + & - \\
\hline McVey ${ }^{*}, 1989, \mathrm{RCT}$ & + & + & + & + & + & + & - & + & - \\
\hline Gayton, 1987, CCT & - & - & + & + & $?$ & + & - & - & + \\
\hline Hogan, 1987, RCT & + & $?$ & + & + & $?$ & + & - & - & + \\
\hline Fretwell, 1990, RCT & $?$ & $?$ & + & + & $?$ & $?$ & + & $?$ & + \\
\hline Hogan, 1990, CCT & - & $?$ & $?$ & $?$ & $?$ & - & - & - & + \\
\hline Inouye, 1993, CCT & - & + & + & - & + & + & $?$ & + & + \\
\hline Thomas, 1993, RCT & + & + & + & + & $?$ & $?$ & - & $?$ & + \\
\hline Winograd, 1993, RCT & + & + & + & + & + & + & - & - & + \\
\hline Clark, 1995, CCT & - & - & - & - & $?$ & - & $?$ & - & - \\
\hline Landefeld, 1995, RCT & + & + & - & + & $?$ & - & - & $?$ & + \\
\hline Reuben, 1995, RCT & + & $?$ & + & + & ? & $?$ & - & + & + \\
\hline Asplund, 2000, RCT & + & + & + & + & $?$ & - & - & $?$ & - \\
\hline Counsell, 2000, RCT & + & + & + & - & + & - & - & + & + \\
\hline Cohen $^{\dagger}$, 2002, RCT & + & + & + & + & ? & + & - & + & + \\
\hline Phibbs $^{\dagger}$, 2006, RCT & + & + & + & + & $?$ & + & - & + & + \\
\hline Basic, 2005, RCT & + & + & + & - & + & + & $?$ & + & + \\
\hline
\end{tabular}

Note $:+=$ yes $;-=$ no; $?=$ unclear $/$ not reported

*same study; ${ }^{\dagger}$ same study 


\section{Reference List}

(1) Podrazik PM, Whelan CT. Acute Hospital Care for the Elderly Patient: Its Impact on Clinical and Hospital Systems of Care. Med Clin North Am 2008 March;92(2):387406.

(2) Van Craen K, Braes T, Wellens $\mathrm{N}$ et al. The effectiveness of inpatient geriatric evaluation and management units: a systematic review and meta-analysis. $J \mathrm{Am}$ Geriatr Soc 2010 January;58(1):83-92.

(3) Ellis G, Langhorne P. Comprehensive geriatric assessment for older hospital patients. Br Med Bull 2005;71(1):45-59.

(4) Baztan JJ, Suarez-Garcia FM, Lopez-Arrieta J, Rodriguez-Manas L, RodriguezArtalejo F. Effectiveness of acute geriatric units on functional decline, living at home, and case fatality among older patients admitted to hospital for acute medical disorders: meta-analysis. BMJ 2009 January 22;338:b50.

(5) Covinsky KE, Palmer RM, Fortinsky RH et al. Loss of Independence in Activities of Daily Living in Older Adults Hospitalized with Medical Illnesses: Increased Vulnerability with Age. J Am Geriatr Soc 2003 March 26;51(4):451-8.

(6) Boyd CM, Landefeld CS, Counsell SR et al. Revovery of Activities of Daily Living in Older Adults After Hospitalization for Acute Medical Illness. J Am Geriatr Soc 2008 November 20;56(12):2171-9.

(7) Inouye SK, Schlesinger MJ, Lydon TJ. Delirium: a symptom of how hospital care is failing older persons and a window to improve quality of hospital care. Am J Med 1999 May;106(5):565-73.

(8) Healey F, Scobie S, Oliver D, Pryce A, Thomson R, Glampson B. Falls in English and Welsh hospitals: a national observational study based on retrospective analysis of 12 months of patient safety incident reports. Qual Saf Health Care 2008 December;17(6):424-30.

(9) Zeegers M, De Bruijne MC, Wagner C et al. Adverse events and potentially preventable deaths in Dutch hospitals: results of a retrospective patient record review study. Qual Saf Health Care 2009 June 14;18:297-302.

(10) Hofmarcher MM, Oxley H, Rusticelli E. Improving Health System Performance Through Better Care Coordination. OECD; 2007 Dec 12. Report No.: 30.

(11) Hogan DB, Fox RA, Badley BW, Mann OE. Effect of a geriatric consultation service on management of patients in an acute care hospital. CMAJ 1987 April 1;136(7):7137.

(12) Hogan DB, Fox RA. A Prospective Controlled Trial of a Geriatric Consultation Team in an Acute-care Hospital. Age Ageing 1990 March;19(2):107-13.

(13) Winograd CH, Gerety MB, Lai NA. A Negative Trial of Inpatient Geriatric Consultation: Lessons Learned and Recommendations for Future Research. Arch Intern Med 1993 September 13;153(17):2017-23. 
(14) Clark LR, Fraaza V, Schroeder S, Maddens ME. Alternative Nursing Environments: Do They Affect Hospital Outcomes? J Gerontol Nurs 1995 November;21(11):32-8.

(15) Reuben DB, Borok GM, Wolde-Tsadik G et al. A Randomized Trial of Comprehensive Geriatric Assessment in the Care of Hospitalized Patients. N Engl J Med 1995 May 18;332(20):1345-50.

(16) Cohen HJ, Feussner JR, Weinberger M et al. A Controlled Trial of Inpatient and Outpatient Geriatric Evaluation and Management. N Engl J Med 2002 March 21;346(12):905-12.

(17) Basic D, Conforti DA. A prospective, randomised controlled trial of an aged care nurse intervention within the Emergency Department. Aust Health Rev 2005 February;29(1):51-9.

(18) Phibbs CS, Holty JE, Goldstein MKM et al. The Effect of Geriatrics Evaluation and Management on Nursing Home Use and Health Care Costs: Results From a Randomized Trial. Med Care 2006 January;44(1):91-5.

(19) Collard AF, Bachman SS, Beatrice DF. Acute Care Delivery for the Geriatric Patient: An Innovative Approach. QRB Qual Rev Bull 1985 June;11(6):180-5.

(20) Landefeld CS, Palmer RM, Kresevic DM, Fortinsky RH, Kowal J. A Randomized Trial of Care in a Hospital Medical Unit Especially Designed to Improve the Functional Outcomes of Acutely Ill Older Patients. N Engl J Med 1995 May $18 ; 332(20): 1338-44$.

(21) Counsell SR, Holder CM, Liebenauer LL et al. Effects of a Multicomponent Intervention on Functional Outcomes and Process of Care in Hospitalized Older Patients: A Randomized Controlled Trial of Acute Care for Elders (ACE) in a Community Hospital. J Am Geriatr Soc 2000 December;48(12):1572-81.

(22) Fretwell MD, Raymond PM, McGarvey ST et al. The Senior Care Study. A Controlled Trial of a Consultative/Unit-Based Geriatric Assessment Program in Acute Care. J Am Geriatr Soc 1990 October;38(10):1073-81.

(23) Inouye SK, Wagner R, Acampora D, Horwitz RI, Cooney LM, Tinetii ME. A Controlled Trial of a Nursing-Centered Intervention in Hospitalized Elderly Medical Patients: The Yale Geriatric Care Program. J Am Geriatr Soc 1993 December;41(12):1353-60.

(24) Gayton D, Wood-Dauphinee S, de Lorimer M, Tousignant P, Hanley J. Trial of a Geriatric Consultation Team in an Acute Care Hospital. J Am Geriatr Soc 1987 August;35(8):726-36.

(25) Thomas DR, Brahan R, Haywood BP. Inpatient Community-Based Geriatric Assessment Reduces Subsequent Mortality. J Am Geriatr Soc 1993 February;41(2):101-4.

(26) Asplund K, Gustafson Y, Jacobsson C et al. Geriatric-Based versus General Ward for Older Acute Medical Patients: a Randomized Comparison of Outcomes and Use of Resources. J Am Geriatr Soc 2000 November;48(11):1381-8. 
(27) Campion EW, Jette A, Berkman B. An Interdisciplinary Geriatric Consultation Service: A Controlled Trial. J Am Geriatr Soc 1983 December;31(12):792-6.

(28) Becker PM, McVey LJ, Saltz CC, Feussner JR, Cohen HJ. Hospital-Acquired Complications in a Randomized Controlled Clinical Trial of a Geriatric Consultation Team. JAMA 1987 May 1;257(17):2313-7.

(29) Centraal Bureau voor de Statistiek. Sterfte na 1e ziekenhuisopname, naar geslacht, leeftijd en Beldo-diagnose. 26-2-2008. Den Haag/Heerlen, CBS.

(30) Boyd CM, Ricks M, Fried LP et al. Functional Decline and Recovery of Activities of Daily Living in Hospitalized, Disabled Older Women: The Women's Health and Aging Study I. J Am Geriatr Soc 2009 October;57(10):1757-66.

(31) McVey LJ, Becker PM, Saltz CC, Feussner JR, Cohen HJ. Effect of a Geriatric Consultation Team on Functional Status of Elderly Hospitalized Patients. A Randomized, Controlled Clinical Trial. Ann Intern Med 1989 January 1;110(1):79-84.

(32) Landefeld CS. Improving health care for older persons. Ann Intern Med 2003 September 2;139:421-4.

(33) Palmisano-Mills C. Common Problems in Hospitalized Older Adults. J Gerontol Nurs 2007 January;33(1):48-54.

(34) Parker G, Bhakta P, Katbamna S et al. Best place of care for older people after acute and during subacute illness: a systematic review. J Health Serv Res Policy 2000 July;5(3):176-89.

(35) Harari D, Martin FC, Buttery A, O'Neill S, Hopper A. The older persons' assessment and liaison team 'OPAL': evaluation of comprehensive geriatric assessment in acute medical inpatients. Age Ageing 2007 July 26;36:670-5.

(36) Tucker D, Bechtel G, Quartana C et al. The OASIS Program: Redesigning Hospital Care for Older Adults. Geriatr Nurs 2003 March;27(2):112-7.

(37) Inouye SK, Bogardus ST Jr, Baker DI, Leo-Summers L, Cooney LM Jr. The Hospital Elder Life Program: a model of care to prevent cognitive and functional decline in older hospitalized patients. Hospital Elder Life Program. J Am Geriatr Soc 2000 December;48(12):1697-706.

(38) Rubin FH, Williams JT, Lescisin DA, Mook WJ, Hassan S, Inouye SK. Replicating the Hospital Elder Life Program in a Community Hospital and Demonstrating Effectiveness Using Quality Improvement Methodology. J Am Geriatr Soc 2006;54:969-74.

(39) Guthrie PF, Edinger G, Schumacher S. TWICE: A NICHE program at North Memorial Health Care. Geriatr Nurs 2002 May;23(3):133-8.

(40) Lyons DL, Blum T, Curtin PM, Rimley SM. Improving care of the older adult. Medsurg Nurs 2006 June;15(3):176-7-181. 
(41) Jayadevappa R, Bloom BS, Raziano DB, Lavizzo-Mourey R. Dissemination and Characteristics of Acute Care for Elders (ACE) Units in the United States. Int J Technol Assess Health Care 2003;19(1):220-7.

(42) Covinsky KE, Palmer RM, Kresevic DM et al. Improving Functional Outcomes in Older Patients: Lessons from an Acute Care for Elders Unit. Jt Comm J Qual Improv 1998 February;24(2):63-76.

(43) Sennour Y, Counsell S, Jones J, Weiner M. Development and Implementation of a Proactive Geriatrics Consultation Model in Collaboration with Hospitalists. J Am Geriatr Soc 2009 November;57(11):2139-45.

(44) Malone M, Vollbrecht M, Stephenson J, Burke L, Pagel P, Goodwin J. Acute Care for Elders (ACE) tracker and e-Geriatrician: methods to disseminate ACE concepts to hospitals with no geriatricians on staff. J Am Geriatr Soc 2010 January;58(1):161-7.

(45) Craig P, Dieppe P, Macintyre S, Mitchie S, Nazareth I, Petticrew M. Developing and evaluating complex interventions: the new Medical Research Council guidance. BMJ 2008 October 25;337:979-83.

(46) McDaniel RR, Lanham HJ, Anderson RA. Implications of complex adaptive systems theory for the design of research on health care organizations. Health Care Manage Rev 2009;34(2):191-9.

(47) Wenger NS, Shekelle PG, ACOVE Investigators. Quality Indicators for Assessing Care of Vulnerable Elders: Assessing Care of Vulnerable Elders: ACOVE Project Overview. Ann Intern Med 2001 October 16;135(8):642-6.

(48) Arora VM, Johnson M, Olson J et al. Using assessing care of vulnerable elders quality indicators to measure quality of hospital care for vulnerable elders. $J$ Am Geriatr Soc 2007 November;55(11):1705-11.

(49) Campbell S, Seymour D, Primrose W, ACMEPLUS Project. A systematic literature review of factors affecting outcome in older medical patients admitted to hospital. Age Ageing 2004 March;33(2):110-5.

(50) Porter ME. What Is Value in Health Care? New England Journal of Medicine 2010 December 8;363(26):2477-81.

(51) Saltz CC, McVey LJ, Becker PM, Feussner JR, Cohen HJ. Impact of a Geriatric Consultation Team on Discharge Placement and Repeat Hospitalization. Gerontologist 1988 June;28(3):344-50. 


\section{SUPPLEMENTARY ONLINE CONTENT}

"Effects of hospital-wide interventions to improve care for frail older inpatients: a systematic review"

Appendix A - Search Strategy Pubmed 2

Appendix B - Search Strategy Cochrane Library 3

Appendix C - Search Strategy Cinahl 4

Appendix D - Data Abstraction Form 5

Appendix E - Results Effectiveness of Interventions of included studies $\quad 15$

$\begin{array}{lr}\text { Appendix F - Table } 1 \text { Characteristics of interventions } & 18\end{array}$

Appendix G - Table 2 Detailed results of included studies 25

Appendix H - Table 3 Discussion points of included studies 30 


\section{Appendix A Search strategy Pubmed}

$\begin{array}{ll}\text { Search strategy: } & \text { created by FB } \\ \text { Date search: } & \text { 15 May 2009 } \\ \text { Limits: } & \text { English, Dutch } \\ & 1980-2009 \\ \text { Hits: } & 800 \\ \text { Initial selection: } & 69 \text { by FB \& SR }\end{array}$

Search Strategy:

1. aged [mesh]

2. frail [tiab]

3. geriatric [tiab]

4. elderly [tiab]

5. elder [tiab]

6. older [tiab]

7. $\mathrm{OR} / 1-7$

8. hospital* $[\mathrm{tw}]$

9. "health services for the aged" $[\mathrm{tw}]$

10. "delivery of health care, integrated" [tw]

11. "comprehensive health care" [tw]

12. "patient-centered care" [tw]

13. "geriatric assessment" [tw]

14. "geriatric care" [tiab]

15. OR/9-14

16. "quality Assurance, health care" [tw]

17. "total quality management" [tw]

18. "outcome and process assessment (health care)" [mesh]

19. "health services research" [mesh]

20. "program development" [tw]

21. "program evaluation" $[\mathrm{tw}]$

22. "organizational innovation" $[\mathrm{tw}]$

23. benchmarking $[\mathrm{tw}]$

24. OR/16-23

25. "randomized controlled trial" [pt]

26. random* $[\mathrm{tw}]$

27. control* $[\mathrm{tw}]$

28. intervention? $[\mathrm{tw}]$

29. evaluat* $[\mathrm{tw}]$

30. OR/25-29

31. animal/

32. human/

33. 31 NOT (31 AND 32)

34. 30 NOT 33

35. 7 AND 8 AND 15 AND 24 AND 34 Limits: Publication Date from 1980, English, Dutch 


\section{Appendix B Search Strategy Cochrane Library}

$\begin{array}{ll}\text { Search strategy: } & \text { created by FB } \\ \text { Date search: } & \text { 19 May 2009 } \\ \text { Limits: } & 1980-2009 \\ \text { Hits: } & 193 \\ \text { Initial selection: } & 21 \text { by FB \& SR } \\ \text { Not in Pubmed: } & 4\end{array}$

\section{Search Strategy:}

1. MeSH descriptor Aged explode all trees

2. (frail OR geriatric OR elderly OR elder OR older): ti,ab,kw, from 1980 to 2009

3. $\mathrm{OR} / 1-2$

4. MeSH descriptor Hospitals explode all trees

5. hospital*: ti,ab,kw, from 1980 to 2009

6. OR/4-5

7. MeSH descriptor Health Services for the Aged explode all trees

8. MeSH descriptor Delivery of Health Care, Integrated, this term only

9. MeSH descriptor Comprehensive Health Care, this term only

10. MeSH descriptor Patient-Centered Care, this term only

11. MeSH descriptor Geriatric Assessment, this term only

12. "geriatric assessment": ti,ab,kw, from 1980 to 2009

13. "integrated care": ti,ab,kw, from 1980 to 2009

14. "integrated services": ti,ab,kw, from 1980 to 2009

15. OR/7-14

16. MeSH descriptor Quality Assurance, Health Care, this term only

17. MeSH descriptor Total Quality Management, this term only

18. MeSH descriptor Outcome and Process Assessment (Health Care) explode all trees

19. MeSH descriptor Health Services Research explode all trees

20. MeSH descriptor Program Evaluation explode all trees

21. MeSH descriptor Program Development explode all trees

22. MeSH descriptor Organizational Innovation explode all trees

23. "program development": ti,ab,kw, from 1980 to 2009

24. "program evaluation": ti,ab,kw, from 1980 to 2009

25. "program AND innovation": ti,ab,kw, from 1980 to 2009

26. "organi*ation AND innovation": ti,ab,kw, from 1980 to 2009

27. OR/16-26

28. \#3 AND \#6 AND \#15 AND \#27, from 1980 to 2009 


\section{Appendix C Search Strategy CINAHL}

$\begin{array}{ll}\text { Search strategy: } & \text { created by FB } \\ \text { Date search: } & \text { 19 May 2009 } \\ \text { Limits: } & \text { English, Dutch } \\ & 1980-2009 \\ & 182 \\ \text { Hits: } & 16 \text { by FB \& SR } \\ \text { Initial selection: } & 6\end{array}$

\section{$\underline{\text { Search Strategy }}$}

1. $\mathrm{MH}$ "Aged + "

2. TI frail OR AB frail

3. TI geriatric $\mathrm{OR} A B$ geriatric

4. TI elderly OR AB elderly

5. TI elder OR AB elder

6. Ti older OR AB older

7. $\mathrm{OR} / 1-6$

8. MH "Hospitals + "

9. TI hospital* OR AB hospital*

10. OR/8-9

11. MH "Health Services for the Aged"

12. MH "Health Care Delivery, Integrated"

13. MH "Patient Centered Care"

14. MH "Geriatric Assessment"

15. $\mathrm{MH}$ "Geriatrics")

16. TI "geriatric assessment" OR AB "geriatric assessment"

17. $\mathrm{MH}$ "Gerontologic Care"

18. OR/11-17

19. MH "Quality Improvement"

20. MH "Benchmarking"

21. MH "Outcome Assessment"

22. MH "Process Assessment (Health Care)"

23. MH "Program Development"

24. MH "Program Evaluation"

25. MH "Health Services Research+"

26. AB innovation

27. $\mathrm{AB}$ benchmarking

28. (AB hospital AND AB program AND AB development)

29. (AB hospital AND AB program AND AB evaluation)

30. OR/19-29

31. MH "Clinical Trials"

32. TX control* $^{*}$

33. TX random*

34. MH "Comparative Studies"

35. TX experiment*

36. TX (time N5 series)

37. TX impact

38. TX intervention*

39. TX evaluat*

40. TX effect?

41. MH "Pretest-Posttest Design+"

42. $\mathrm{MH}$ "Quasi-Experimental Studies+"

43. OR/31-42

44. JN "cochrane database of systematic reviews"

45. 43 NOT 44

46. 7 AND 10 AND 18 AND 30 AND 45: Limiters - Published Date from: 1980-01/2009-12; Language Dutch, English 
Cochrane Effective Practice and Organisation of Care Group (EPOC)

\section{Data Abstraction Form}

This form can be used to record the results of data extraction and is intended for use in conjunction with the EPOC Data Collection Checklist. EPOC scope: The effect(s) of a behavioural/educational, financial, organisational or regulatory intervention(s) is evaluated.

\section{Data collection}

Name of reviewer:

Date:

Study reference:

\section{Inclusion criteria specifically for this review}

1) Population

YES/NO

- Age 65 or older:

- Inpatient:

- ((Assessment of) frailty: ...

2) Setting

- Hospital:

3) Intervention

YES/NO

- Hospital wide:

- Aim to improve either quality, efficiency

or (cost-)effectiveness of care for frail

inpatients aged 65 or more:

- Organisational (structure or process)

4) Outcome (at least one of the following)

- Quality of care

- Patient safety

- Patient/proxy related outcomes

- Resource use

- Costs

\section{Inclusion criteria}

\subsection{Study design}

\subsubsection{RCT designs}

\subsubsection{CCT designs}

\subsubsection{CBA designs}

a) Contemporaneous data collection

$\square$ DONE
$\square$ NOT CLEAR $\rightarrow \quad$ contact editor
$\square$ NOT DONE


b) Appropriate choice of control site/activity

$\square$ DONE

$\square$ NOT CLEAR $\rightarrow \quad$ contact editor

$\square$ NOT DONE

c) Number of sites: Studies using second site as controls

$\square$ DONE

$\square$ NOT DONE

\subsubsection{ITS designs}

a) Clearly defined point in time when the intervention occurred

$\square$ DONE

$\square$ NOT CLEAR $\rightarrow \quad$ contact editor .............

$\square$ NOT DONE

b) At least 3 data points before and 3 after the intervention

$\square$ DONE

$\square$ NOT CLEAR $\rightarrow \quad$ contact editor

$\square$ NOT DONE

\subsection{Methodological inclusion criteria}

a) The objective measurement of performance/provider behaviour or health/patient outcomes in a clinical not test situation

$\square$ DONE

$\square$ NOT CLEAR $\rightarrow \quad$ contact editor

$\square$ NOT DONE

b) Relevant and interpretable data presented or obtainable $\square$ DONE

$\square$ NOT CLEAR $\rightarrow \quad$ contact editor

$\square$ NOT DONE

N.B. A study must meet the minimum criteria for EPOC scope, design, and methodology for inclusion in EPOC reviews. If it does not, COLLECT NO FURTHER DATA. 


\section{Interventions}

\subsection{Type of intervention}

(state all interventions for each comparison/study group)

\section{$\square$ Provider orientated}

$\square$ Revision of professional roles

$\square$ Clinical multidisciplinary teams

$\square$ Formal integration of services

$\square$ Skill mix changes (changes in numbers, types or qualifications of staff)

$\square$ Continuity of care (arrangements for follow-up, case management)

$\square$ Satisfaction of providers with the conditions of work and the material and psychic rewards (e.g. interventions to 'boost morale')

$\square$ Communication and case discussion between health professionals

\section{$\square$ Other}

\section{Structural}

$\square$ Changes to the setting/site of service delivery

$\square$ Changes in physical structure, facilities and equipment

$\square$ Changes in medical records system

$\square$ Changes in scope and nature of benefits and services

$\square$ Presence and organisation of quality monitoring mechanisms

$\square$ Ownership, accreditation, and affiliation status of hospitals

$\square$ Staff organisation

$\square$ Other

\section{Group 1:}

Group 2:

\section{Group 3:}

Group 4:

\subsection{Control(s)}

no intervention control group

standard practice control group (if different to (a) above)

untargeted activity

other (e.g. another intervention);

\section{Type of Targeted Behaviour}

\section{(state more than one where appropriate)}

clinical prevention services

test ordering

procedures

general management of a problem professional-patient communication

financial (resource use)

patient outcome

other (e.g. another intervention); diagnosis

referrals

prescribing

patient education/advice

record keeping

discharge planning

NOT CLEAR 


\section{Participants}

\subsection{Characteristics of Participating Providers}

\subsubsection{Profession(s)}

physicians

pharmacists

psychologists

mixed;

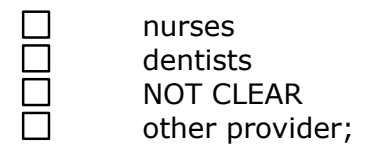

\subsubsection{Clinical specialty}

Emergency Department

NOT APPLICABLE

NOT CLEAR

\subsection{Characteristics of Participating Patients}

4.2.1 Clinical problem (State the area(s) that the intervention targets)

NOT CLEAR

\subsubsection{Other patient characteristics}
a) Age (mean \& range)
$\square \quad$ Intervention group:
$\square \quad$ Comparison group:
$\square \quad$ NOT CLEAR
b) Gender (distribution)
Intervention group:
Comparison group:
NOT CLEAR
c) Admission
Emergency Department
Acute but no representation in ED
Planned
NOT CLEAR
d) Other (specify: e.g. frailty, multimorbidity, first hospital admission)

\subsubsection{Number of patients included in the study}

a) Episodes of care

$\square \quad$ NOT CLEAR 

b) Patients (intervention \& control)
Intervention group:
Comparison group:
NOT CLEAR
c) Hospitals
NOT CLEAR

\section{Setting}

\subsection{Reimbursement system}
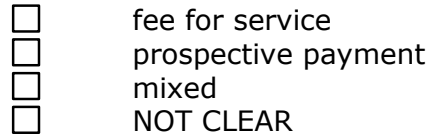
$\square \quad$ global budget
capitation
other:

\subsection{Location of Care}

$\square \quad \begin{aligned} & \text { Inpatient only } \\ & \square \quad \text { Mixed (in- \& outpatient care) }\end{aligned}$

\subsection{Academic status}

$$
\begin{aligned}
& \square \quad \text { University based/Teaching hospital } \\
& \text { Non-teaching hospital } \\
& \text { NOT CLEAR }
\end{aligned}
$$

\subsection{Country}

NOT CLEAR

\subsection{Proportion of eligible providers (or allocation units)}

NOT CLEAR

\section{Methods}

\subsection{Unit of allocation (i.e. who or what was allocated to study groups)}

NOT CLEAR

\subsection{Unit of analysis (i.e. results analysed as events per practice)}

$$
\text { NOT CLEAR }
$$

6.3 Power calculation (reporting power, clinical significance, statistical significance and $N$ )

$$
\square \text { DONE }
$$

$\square$ NOT CLEAR

$\square$ NOT DONE 


\subsection{Quality criteria $\rightarrow=$ risk of bias DRAFT 2009}

\subsubsection{Risk of bias for studies with a separate control group}

(RCTS, CCTS, CBAs)

a) Was the allocation sequence adequately generated?

$\square$ YES

$\square$ NO

$\square$ UNCLEAR

b) Was the allocation adequately concealed?

$\square$ YES

$\square$ NO

$\square$ UNCLEAR

c) Were baseline outcome measures similar?*

$\square$ YES

$\square$ NO

$\square$ UNCLEAR

d) Were baseline characteristics similar?

$\square$ DONE

$\square$ NOT CLEAR

$\square$ NOT DONE

e) Were incomplete outcome data adequately addressed?*

$\square$ YES

$\square$ NO

$\square$ UNCLEAR

f) Was knowledge of the allocated interventions adequately prevented during the study?*

$\square$ YES

$\square$ NO

$\square$ UNCLEAR

g) Was the study adequately protected against contamination?

$\square$ YES

$\square$ NO

$\square$ UNCLEAR

h) Was the study free from selective outcome reporting?

$\square$ YES

$\square$ NO

$\square$ UNCLEAR

i) Was the study free from other risks of bias?

$\square$ YES

$\square$ NO 


\subsubsection{Risk of bias for interrupted time series studies}

a) Was the intervention independent of other changes?

$\square$ YES

$\square$ NO

b) Was the shape of the intervention effect pre-specified?

$\square$ YES

$\square$ NO

c) Was the intervention unlikely to affect data collection?

$\square$ YES

$\square$ NO

d) Was knowledge of the allocated interventions adequately prevented during the study?***

$\square$ YES

$\square$ NO

$\square$ UNCLEAR

e) Were incomplete outcome data adequately addressed?***

$\square$ YES

$\square$ NO

$\square$ UNCLEAR

f) Was the study free from selective outcome reporting?

$\square$ YES

$\square$ NO

$\square$ UNCLEAR

g) Was the study free from other risks of bias?

$\square$ YES

$\square$ NO

\subsubsection{Consumer involvement}

$\square$ DONE

$\square$ NOT CLEAR

$\square$ NOT DONE

\section{Prospective identification by investigators of} barriers to change

(Identification of specific barriers to change in the target population, which were addressed by the intervention, e.g. information management, clinical uncertainty, sense of competence, patient expectations, standards of practice, financial disincentives, administrative constraints, etc.)

$\square$ DONE:

$\square$ NOT DONE

NOT CLEAR 


\section{Intervention}

\subsection{Characteristics of the intervention}

a) Evidence base of recommendation

$\square$ DONE

$\square$ NOT CLEAR

$\square$ NOT DONE

b) Purpose of recommendations

$\square$ Appropriate management

$\square$ Cost containment

$\square$ Other

$\square$ NOT CLEAR

\subsection{Source}

$$
\text { NOT CLEAR }
$$

\subsection{Intervention based upon implementation of clinical practice}

guidelines (i.e. based upon clear recommendations for practice)

$\square$ DONE

$\square$ NOT CLEAR

$\square$ NOT DONE

\subsection{Recipient}
$\square$ Individual
$\square$ Group
$\square$ NOT CLEAR

8.8 Deliverer (e.g. pharmacist, local expert, research worker, management representative, computer)

$$
\text { NOT CLEAR }
$$

\subsection{Timing}

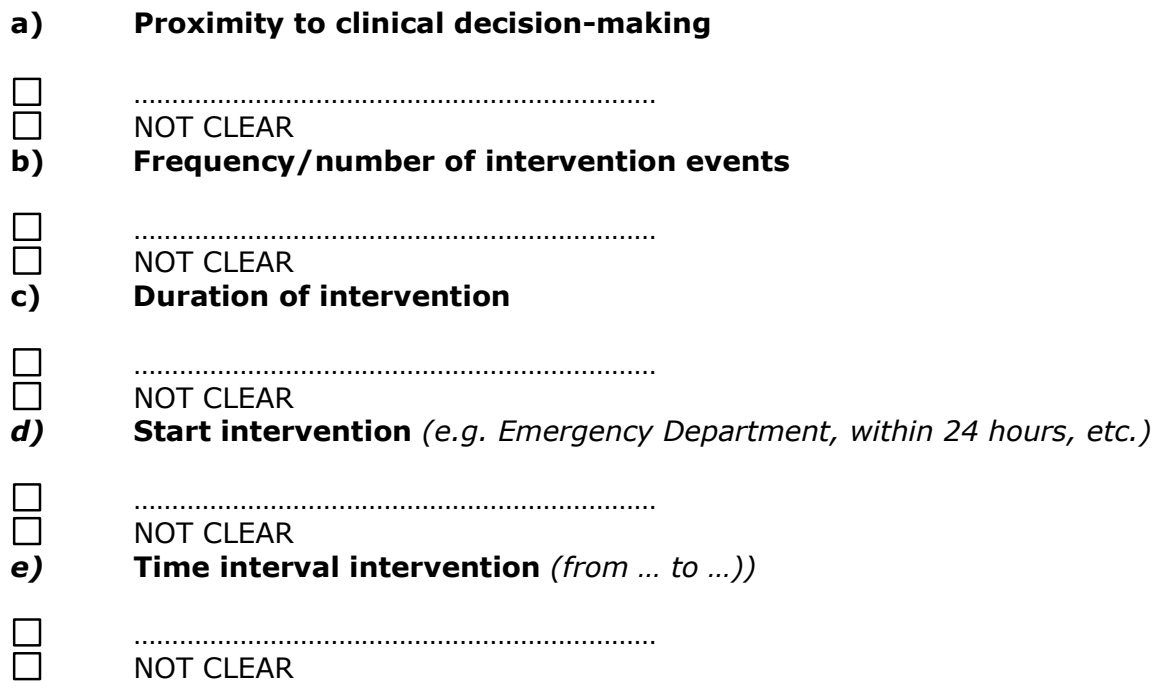




\subsection{Source of funding}

$\square \quad$ NOT CLEAR

\subsection{Ethical approval}

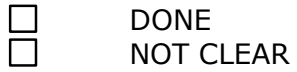

\section{Outcomes}

\subsection{Description of the main outcome measure(s).}

a) Health professional outcomes/process measures

$\square \quad$ NOT CLEAR

b) Patient outcomes

$\square \quad$ NOT CLEAR

c) Economic variables

- Costs of the intervention

$\square$ DONE:

$\square$ NOT DONE

- Changes in direct health care costs as a result of the intervention

$\square$ DONE:

$\square$ NOT DONE

- Changes in non-health care costs as a result of the intervention

$\square$ DONE:

$\square$ NOT DONE

- Costs associated with the intervention are linked with provider or patient outcomes in an economic evaluation

$\square$ DONE:

$\square$ NOT CLEAR

$\square$ NOT DONE

9.2 Length of time during which outcomes were measured after initiation of the intervention.

$\square \quad$ NOT CLEAR

\subsection{Length of post- intervention follow-up period.}

$\square$ DONE:

$\square$ NOT CLEAR

$\square$ NOT DONE 


\subsection{Identify a possible ceiling effect:}

a) Identified by investigator

$\square \quad$ Yes

NOT CLEAR

b) Identified by reviewer

$\square \quad$ Yes

NOT CLEAR

\section{Results}

State the results as they will be entered in the review, and describe how these were calculated.

Check the data collection checklist for RCTs, CCTs, CBAs \& ITSs items to be considered.

\section{Additional}

Key conclusions of the study authors:

Points of discussion by study author:

Points of discussion by review author:

References to other relevant studies:

Correspondence required:

Citation and contact details:

In case of exclusion, note reason: 


\section{APPENDIX E Results Effectiveness of Interventions}

\section{Effectiveness of Interventions}

Primary outcomes were functional performance, length of stay, mortality, discharge destination, readmission, complications, resource use and costs (further details: Appendix Table 2).

\section{Functional outcomes}

Fourteen studies presented results on functional patient outcomes. Of these, five studies showed significant effects for patients in the intervention group in mental health, emotional or cognitive status. A geriatric consultation team (GCT) for all patients $\geq 75$ years hospital-wide resulted in a larger improvement in mental health status (scale 0-10, measure not further specified) at discharge (I vs C: 1.5 vs $0.8, \mathrm{p} \leq .01, \mathrm{~d}=.25$ ). ${ }^{1} \mathrm{~A}$ GCT throughout the hospital including only frail males $\geq 65$ years resulted in a higher Mini-Mental State Examination (MMSE) score at one year follow-up (I vs C: 24.3 vs 21.4, p=.02, d=.35). ${ }^{2}$ A GCT in a Health Maintenance Organization (HMO) including frail patients $\geq 65$ years resulted in a higher mental health index score at 3 months follow-up (I vs C: 71.6 (95\%CI 70.3-72.9) vs 69.5 (95\%CI 68.0-71.0), p=.04; scale details: Appendix Table 2), and a higher score on a current health perceptions scale at 12 months follow-up (I vs C: 50.1 (95\% CI 48.1-52.1) vs 46.3 (95\%CI 44.0-48.6), $\mathrm{p}=.01){ }^{3,4}$ A GCT attached to a hospital ward for all patients $\geq 75$ years resulted in a higher change in emotional status from baseline to six weeks following discharge for the intervention group (Self Rating Depression Scale, $\mathrm{p}=.045$ ). ${ }^{4} \mathrm{~A}$ dayroom for patients $\geq 65$ years and at risk of falls and mental status changes resulted in a higher mean mental status score (more mentally impaired) at discharge (I vs C: Short Portable Mental Status Questionnaire score 5.65 vs 3.42, $\mathrm{p}<.05){ }^{5}$

Three studies demonstrated significant improvements in physical outcomes. An Acute Care for Elders (ACE) unit meant for all older patients resulted in greater improvements in ability to perform Activities of Daily Living (ADL) from admission to discharge (Katz index scale I vs C: $\mathrm{p}=.009){ }^{6}$ A hospital-wide GCT for frail patients $\geq 75$ years showed greater improvement in physical functioning at 12 months follow-up (I vs C: increased Barthel index $75 \%$ vs $44 \%, \mathrm{p}<.01$ ). ${ }^{7}$ A GCT throughout the hospital for frail (male) patients $\geq 65$ years showed a higher mean (positive) change in score in bodily pain at 12 months follow-up (I vs C: 24.0 vs 20.0, on the Medical Outcomes Study 36-Item Short-Form General Health Survey (SF-36); $\mathrm{p}=.01){ }^{8}$ 


\section{Mortality}

Five studies had mortality as one of the primary outcome measures, of which one study introducing a GCT for frail patients $\geq 75$ years throughout the hospital revealed positive results on survival at 6 months follow-up $(\mathrm{p}<.02)^{7}$, and one study introducing a hospital-wide GCT for patients $\geq 70$ years showed a lower mortality rate at 6 months follow-up (I vs C: $6 \%$ vs $21 \%, \mathrm{p}=.01)^{9}$.

\section{Length of Stay}

Of nine studies studying length of stay (LOS), one study which adopted a primary nursing model of care in a ward for all randomized patients $\geq 65$ years had a shorter LOS in one of the two experimental sites (I vs C: 8.7 days vs 10.8 days, $\mathrm{p} \leq .01$ ). ${ }^{10}$

\section{Discharge Destination}

Eight studies focused on discharge destination. Of these, one which adopted a primary nursing model of care in a ward for all patients $\geq 65$ years showed a statistically significantly higher nursing homes admission rate for one of the two experimental sites (I vs C: $19 \%$ vs $17 \%$, $\mathrm{p} \leq .05$ ). ${ }^{10}$ The other study introducing a GCT throughout the hospital for frail (male) patients $\geq 65$ years had a lower number of nursing home admissions at 12 months (I vs C: 127 vs $177, \mathrm{p}=.001, \mathrm{OR}=.65)^{11}$

\section{Resource Use}

Two studies studied in-hospital resource use, of which one introducing a GCT for all patients $\geq 75$ years throughout the hospital showed a higher rate of referral to rehabilitation services such as physical therapy and occupational therapy (I vs C: $44 \%$ vs $21 \%$, p<.025; $18 \%$ vs $0 \%$, p<.005, respectively). ${ }^{1}$

Six studies measured post-discharge resource use. A GCT throughout the hospital for frail (male) patients $\geq 65$ years showed a lower average number of nursing home days per patient at 12 months (I vs C: 21.2 vs $28.4, \mathrm{p}=.003$, d=-.16). ${ }^{11}$ A GCT for all patients $\geq 75$ years hospital-wide showed a higher mean number of referrals to community services (I vs C: 1.3 vs $0.9, \mathrm{p}<.005$. $\mathrm{d}=.098$ ). ${ }^{1}$ One study including a geriatric-based ward for patients $\geq 70$ years resulted in a higher number of outpatient visits per patient to a physical or occupational therapist up to three months follow-up (I vs C: 0.9 vs $0.2, \mathrm{p}=.02) .^{12}$ 


\section{Readmission}

Four studies, including three GCT interventions and one geriatric-based ward, presenting outcomes on rehospitalization showed no significant differences. $^{2,7,12,13}$ One study including a GCT throughout the hospital for all patients $\geq 70$ years showed fewer readmissions per patient at 6 months follow-up (I vs C: 0.3 vs $0.6, \mathrm{p}=.02, \mathrm{~d}=.37$ ). ${ }^{9}$

\section{Complications}

A primary nursing model of care and a GCT registering hospital-acquired complications showed no statistically significant results. ${ }^{10,14}$

\section{Economic Variables}

Four studies evaluated costs of the intervention. ${ }^{4,10-12}$ Of these, one study which adopted a primary nursing model of care in a ward for all patients $\geq 65$ years demonstrated lower costs per day admitted at one experimental site (I vs C: $\$ 364.76$ vs $\$ 399.53$, p $\leq .5$ ) and lower total hospital costs at the other experimental site (I vs C: $\$ 3591.42$ vs $\$ 4155.54, \mathrm{p} \leq .05) .{ }^{10}$ Another study introducing a GCT throughout the hospital for frail (male) patients $\geq 65$ years showed higher total costs of the index hospitalization (I vs C: $\$ 13449$ vs $\$ 10758$, p $=.0001$ ), lower costs after the initial hospital discharge (I vs C: $\$ 22816$ vs $\$ 26533, \mathrm{p}=.03$ ) and lower nursing home costs at 12 months follow-up (I vs C: $\$ 5853$ vs $\$ 7828$, $\mathrm{p}=.002$ ). ${ }^{11}$ 


\begin{tabular}{|c|c|c|c|c|c|c|}
\hline \multicolumn{7}{|c|}{ Appendix F } \\
\hline \multicolumn{7}{|c|}{ Characteristics of interventions of included studies } \\
\hline Study & Intervention type & $\begin{array}{l}\text { Type targeted } \\
\text { behavior }\end{array}$ & $\begin{array}{l}\text { Characteristics } \\
\text { providers }\end{array}$ & $\begin{array}{l}\text { Characteristics } \\
\text { patients; clinical } \\
\text { problem }\end{array}$ & Excluded patients & $\begin{array}{l}\text { Notes \& secondary } \\
\text { outcomes }\end{array}$ \\
\hline Campion & $\begin{array}{l}\text { Provider orientated; } \\
\text { clinical } \\
\text { multidisciplinary team }\end{array}$ & $\begin{array}{l}\text { Providing more } \\
\text { effective medical } \\
\text { care/improving quality } \\
\text { of medical care, } \\
\text { teaching of } \\
\text { geriatrics/increase } \\
\text { awareness of special } \\
\text { needs of elderly } \\
\text { patients }\end{array}$ & $\begin{array}{l}\text { All GCT members had } \\
\text { a major professional } \\
\text { interest and special } \\
\text { training in the care of } \\
\text { the elderly } \\
\text { Main providers: } \\
\text { geriatrician and } \\
\text { geropsychiatrist }\end{array}$ & $\begin{array}{l}\text { Consultation for all } \\
\text { patients in one ward }\end{array}$ & $\begin{array}{l}\text { Patients previously } \\
\text { cared for by a private } \\
\text { physician and those } \\
\text { admitted to the } \\
\text { neurologic intensive } \\
\text { care unit }\end{array}$ & $\begin{array}{l}\text { Study describes } \\
\text { structure and } \\
\text { function of GCT }\end{array}$ \\
\hline Collard & $\begin{array}{l}\text { Provider orientated; } \\
\text { clinical } \\
\text { multidisciplinary } \\
\text { teams \& revision of } \\
\text { professional roles. } \\
\text { Structural; modified } \\
\text { and remodeled } \\
\text { communal dining } \\
\text { area }\end{array}$ & $\begin{array}{l}\text { High-quality cost- } \\
\text { effective care for } \\
\text { elderly population }\end{array}$ & $\begin{array}{l}\text { Staff selected from } \\
\text { existing staff and } \\
\text { trained to participate in } \\
\text { the project. } \\
\text { Main provider: } \\
\text { (primary) nurse }\end{array}$ & $\begin{array}{l}\text { Emphasizing maximum } \\
\text { patient independence; } \\
\text { maintain or enhance } \\
\text { health status of elderly } \\
\text { patients in Geriatric } \\
\text { Special Care Unit }\end{array}$ & $\begin{array}{l}\text { LOS }<48 \mathrm{~h} \\
\text { Transferred from } \\
\text { other wards than IC }\end{array}$ & $\begin{array}{l}\text { Study describes the } \\
\text { program and its } \\
\text { implementation }\end{array}$ \\
\hline
\end{tabular}




\begin{tabular}{|c|c|c|c|c|c|c|}
\hline Study & Intervention type & $\begin{array}{l}\text { Type targeted } \\
\text { behavior }\end{array}$ & $\begin{array}{l}\text { Characteristics } \\
\text { providers }\end{array}$ & $\begin{array}{l}\text { Characteristics } \\
\text { patients; clinical } \\
\text { problem }\end{array}$ & Excluded patients & $\begin{array}{l}\text { Notes \& secondary } \\
\text { outcomes }^{a}\end{array}$ \\
\hline Becker & $\begin{array}{l}\text { Provider orientated; } \\
\text { clinical } \\
\text { multidisciplinary team }\end{array}$ & $\begin{array}{l}\text { Reduce occurrence of } \\
\text { hospital-acquired } \\
\text { complications }\end{array}$ & $\begin{array}{l}\text { Specialized in } \\
\text { geriatrics or special } \\
\text { interest in geriatrics }\end{array}$ & $\begin{array}{l}\text { All patients in one of } \\
\text { three wards (medical, } \\
\text { surgical, psychiatric) }\end{array}$ & $\begin{array}{l}\text { Admitted to IC, LOS } \\
<48 \text {; previously } \\
\text { care from geriatric } \\
\text { service }\end{array}$ & - \\
\hline Saltz & “ & $\begin{array}{l}\text { Optimize patient's } \\
\text { ability to return home } \\
\text { and reduce likelihood of } \\
\text { rehospitalization or } \\
\text { placement in an } \\
\text { institutional setting, by } \\
\text { (in-hospital) follow-up, } \\
\text { arranging ancillary } \\
\text { LTC-services intense } \\
\text { discharge planning. }\end{array}$ & " & " & “ & - \\
\hline McVey & “ & $\begin{array}{l}\text { Improving functional } \\
\text { performance and } \\
\text { preventing functional } \\
\text { decline, by } \\
\text { interdisciplinary } \\
\text { treatment and } \\
\text { rehabilitation. }\end{array}$ & " & " & “ & - \\
\hline Gayton & $\begin{array}{l}\text { Provider orientated; } \\
\text { clinical } \\
\text { multidisciplinary team }\end{array}$ & $\begin{array}{l}\text { Skilled, comprehensive } \\
\text { \& coordinated } \\
\text { assessment, treatment, } \\
\text { rehabilitation and } \\
\text { discharge planning; } \\
\text { provide information and } \\
\text { support for the families; } \\
\text { family involvement in } \\
\text { care process. }\end{array}$ & $\begin{array}{l}\text { Geriatrics, } \\
\text { rehabilitation, roles of } \\
\text { specialist and nurses } \\
\text { purely consultative. } \\
\text { Main providers, most } \\
\text { hours with patients: } \\
\text { rehabilitation staff }\end{array}$ & $\begin{array}{l}\text { All patients in two of } \\
\text { four wards (but not } \\
\text { everyone assessed?) }\end{array}$ & $\begin{array}{l}\text { Transfers from other } \\
\text { floors } \\
\text { Elective admissions } \\
\text { Accepted for social } \\
\text { reasons }\end{array}$ & - Care process: u \\
\hline
\end{tabular}




\begin{tabular}{|c|c|c|c|c|c|c|}
\hline Study & Intervention type & $\begin{array}{l}\text { Type targeted } \\
\text { behavior }\end{array}$ & $\begin{array}{l}\text { Characteristics } \\
\text { providers }\end{array}$ & $\begin{array}{l}\text { Characteristics } \\
\text { patients; clinical } \\
\text { problem }\end{array}$ & Excluded patients & $\begin{array}{l}\text { Notes \& secondary } \\
\text { outcomes }^{a}\end{array}$ \\
\hline $\begin{array}{l}\text { Hogan } \\
(1987)\end{array}$ & $\begin{array}{l}\text { Provider orientated; } \\
\text { clinical } \\
\text { multidisciplinary team }\end{array}$ & $\begin{array}{l}\text { Emphasis on the } \\
\text { management of } \\
\text { functional problems } \\
\text { and discharge } \\
\text { planning. }\end{array}$ & $\begin{array}{l}\text { Geriatrician main } \\
\text { provider }\end{array}$ & $\begin{array}{l}\text { Patients } \geq 75 \text { and } \\
\text { confusional state, } \\
\text { impaired mobility, falls, } \\
\text { urinary in-continence, } \\
\text { polypharmacy, living in } \\
\text { nursing home, or } \\
\text { admission }<3 \text { months }\end{array}$ & $\begin{array}{l}\text { IC, acute } \\
\text { cerebrovascular } \\
\text { accident }\end{array}$ & $\begin{array}{l}\text { - Resource use in- } \\
\text { hospital: }+ \\
\text { - referrals to } \\
\text { community services: } \\
+\end{array}$ \\
\hline McVey & $\begin{array}{l}\text { Provider orientated; } \\
\text { clinical } \\
\text { multidisciplinary team }\end{array}$ & $\begin{array}{l}\text { Improving functional } \\
\text { performance and } \\
\text { preventing functional } \\
\text { decline, by } \\
\text { interdisciplinary } \\
\text { treatment and } \\
\text { rehabilitation. }\end{array}$ & $\begin{array}{l}\text { Specialized in } \\
\text { geriatrics or special } \\
\text { interest in geriatrics }\end{array}$ & $\begin{array}{l}\text { All patients in one of } \\
\text { three wards (medical, } \\
\text { surgical, psychiatric) }\end{array}$ & $\begin{array}{l}\text { Admitted to IC } \\
\text { Previously received } \\
\text { care from the } \\
\text { geriatric service } \\
\text { LOS }<48 \mathrm{~h}\end{array}$ & - \\
\hline Fretwell & $\begin{array}{l}\text { Provider orientated; } \\
\text { clinical } \\
\text { multidisciplinary } \\
\text { team. Continuity of } \\
\text { care: post-discharge } \\
\text { follow-up } \\
\text { Integration of a } \\
\text { psycho-social and } \\
\text { functional orientation } \\
\text { to care within } \\
\text { traditional model of } \\
\text { patient management. }\end{array}$ & $\begin{array}{l}\text { Preventing the decline } \\
\text { or improve the older } \\
\text { patient 's physical, } \\
\text { mental, and emotional } \\
\text { functions, by } \\
\text { assessment initiated } \\
\text { early in patient's stay, } \\
\text { utilizing existing } \\
\text { personnel, and } \\
\text { integration into routine } \\
\text { practice of hospital } \\
\text { staff. }\end{array}$ & $\begin{array}{l}\text { Physician specializing } \\
\text { in geriatrics, who did } \\
\text { not treat patients } \\
\text { directly. Other team } \\
\text { members were directly } \\
\text { involved in the } \\
\text { patient's care. Main } \\
\text { provider: primary } \\
\text { nurse. Main } \\
\text { responsible: attending } \\
\text { physician. }\end{array}$ & $\begin{array}{l}\text { All patients admitted to } \\
\text { medical or surgical } \\
\text { wards }\end{array}$ & $\begin{array}{l}\text { On protocol } \\
\text { treatment } \\
\text { Require coronary or } \\
\text { intensive care at } \\
\text { admission }\end{array}$ & $\begin{array}{l}\text { - Mortality: ns } \\
\text { - Discharge } \\
\text { destination: u }\end{array}$ \\
\hline
\end{tabular}




\begin{tabular}{|c|c|c|c|c|c|c|}
\hline Study & Intervention type & $\begin{array}{l}\text { Type targeted } \\
\text { behavior }\end{array}$ & $\begin{array}{l}\text { Characteristics } \\
\text { providers }\end{array}$ & $\begin{array}{l}\text { Characteristics } \\
\text { patients; clinical } \\
\text { problem }\end{array}$ & Excluded patients & $\begin{array}{l}\text { Notes \& secondary } \\
\text { outcomes }^{a}\end{array}$ \\
\hline $\begin{array}{l}\text { Hogan } \\
(1990)\end{array}$ & $\begin{array}{l}\text { Provider orientated; } \\
\text { clinical } \\
\text { multidisciplinary team } \\
\text { Continuity of care: } \\
\text { post-discharge follow- } \\
\text { up }\end{array}$ & $\begin{array}{l}\text { Emphasis was on } \\
\text { addressing functional } \\
\text { problems and providing } \\
\text { post-discharge follow- } \\
\text { up. }\end{array}$ & $\begin{array}{l}\text { Only person hired for } \\
\text { the intervention was } \\
\text { the nurse coordinator. } \\
\text { Main provider: } \\
\text { geriatrician. Main } \\
\text { responsible: attending } \\
\text { service. }\end{array}$ & $\begin{array}{l}\text { All patients } \geq 75 \text { and } \\
\text { classified into one of } \\
\text { seven }(3,4 \text { or } 5) \\
\text { categories based on a } \\
\text { questionnaire } \\
\text { developed specifically } \\
\text { for the study termed the } \\
\text { Geriatric Status Scale }\end{array}$ & $\begin{array}{l}\text { Admitted to IC } \\
\text { Stroke consultation } \\
\text { team }\end{array}$ & $\begin{array}{l}\text { GCT was a } \\
\text { component of a } \\
\text { comprehensive } \\
\text { geriatric service } \\
\text { including a day } \\
\text { hospital, inpatient } \\
\text { unit, outpatient } \\
\text { clinics and home- } \\
\text { visiting. } \\
\text { - in-hospital resource } \\
\text { use: ns } \\
\text { - LOS: ns }\end{array}$ \\
\hline Inouye & $\begin{array}{l}\text { Provider orientated: } \\
\text { clinical } \\
\text { multidisciplinary } \\
\text { teams, skill mix } \\
\text { changes. } \\
\text { Integrate geriatric } \\
\text { nursing expertise as } \\
\text { part of standard } \\
\text { nursing care. }\end{array}$ & $\begin{array}{l}\text { Prevention functional } \\
\text { decline in elderly } \\
\text { hospitalized patients }\end{array}$ & $\begin{array}{l}\text { Primary nurses } \\
\text { (trained geriatric } \\
\text { resource nurses), } \\
\text { masters prepared } \\
\text { gerontological nurse } \\
\text { specials, geriatricians. } \\
\text { Key intervention } \\
\text { figures: nurses }\end{array}$ & $\begin{array}{l}\text { All patients admitted to } \\
\text { one of the wards, } \\
\text { identified as frail }\end{array}$ & $\begin{array}{l}\text { Unable to participate } \\
\text { in interviews } \\
\text { Discharged }<24 \mathrm{~h}\end{array}$ & $\begin{array}{l}\text { I: renal \& pulmonary } \\
\text { C: cardiology, } \\
\text { oncology, infectious } \\
\text { diseases } \\
\text { - stratified \& } \\
\text { matched analyses: + } \\
\text { - resource use in- } \\
\text { hospital: + }\end{array}$ \\
\hline Thomas & $\begin{array}{l}\text { Provider orientated: } \\
\text { clinical } \\
\text { multidisciplinary } \\
\text { teams }\end{array}$ & Patient outcome & GCT & $\begin{array}{l}\text { All patients } \geq 70 \text { as } \\
\text { target of frailty }\end{array}$ & $\begin{array}{l}\text { Admitted to IC } \\
\text { Terminally ill } \\
\text { Renal hemodialysis } \\
>50 \text { miles from } \\
\text { hospital }\end{array}$ & - \\
\hline Winograd & $\begin{array}{l}\text { Provider orientated: } \\
\text { clinical } \\
\text { multidisciplinary } \\
\text { teams }\end{array}$ & Health outcomes & GCT & $\begin{array}{l}\text { All functionally impaired } \\
\text { patients } \geq 65 \text { with } \\
\text { confusion, ADL } \\
\text { dependence, } \\
\text { polypharmacy, disabling } \\
\text { chronic illness(es), or a } \\
\text { stressed caregiving } \\
\text { system }\end{array}$ & $\begin{array}{l}\text { ADL independent } \\
\text { Permanent nursing } \\
\text { home } \\
\text { Terminal illness with } \\
\text { life expectancy <6 } \\
\text { months }\end{array}$ & - \\
\hline
\end{tabular}




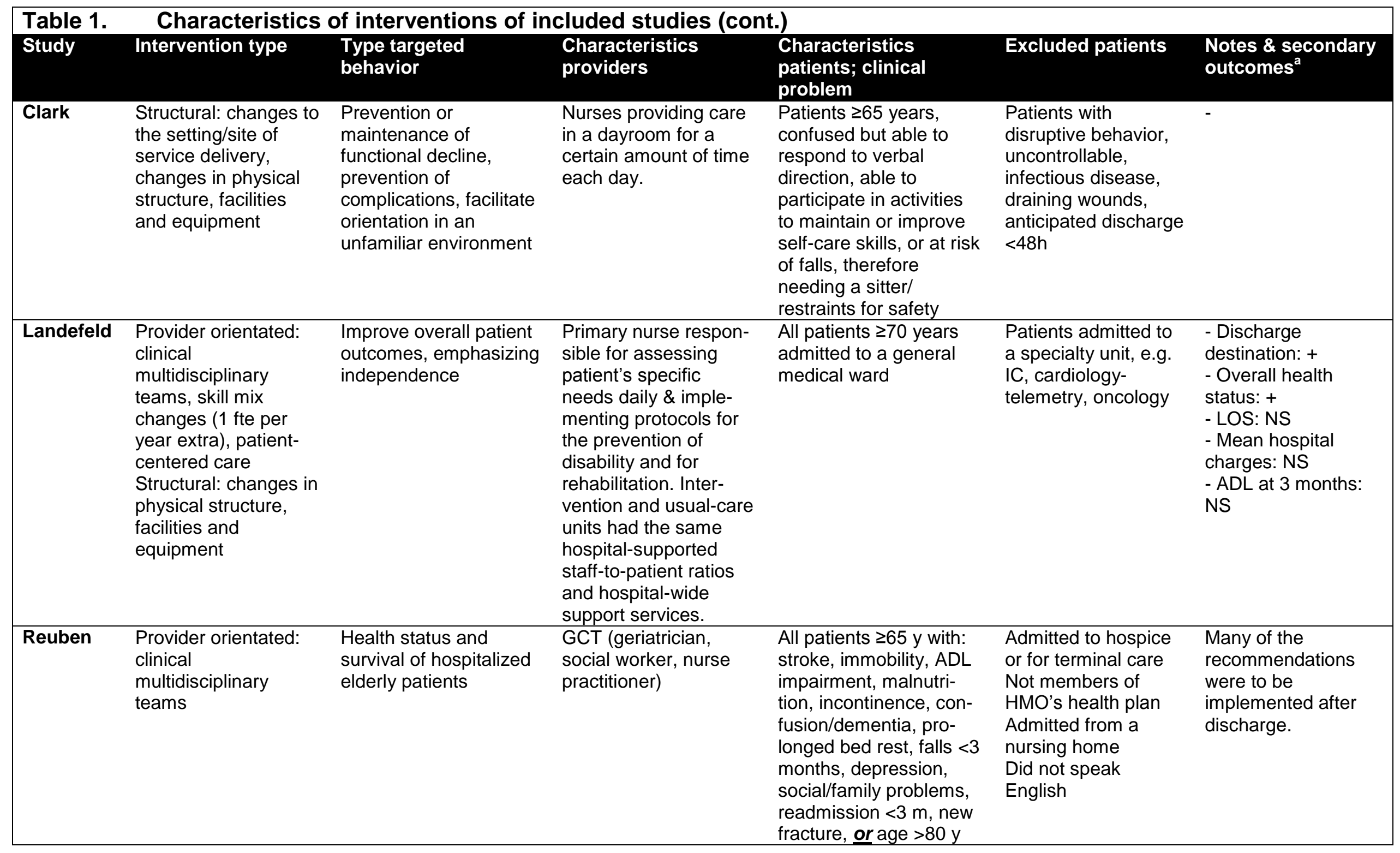




\begin{tabular}{|c|c|c|c|c|c|c|}
\hline Study & Intervention type & $\begin{array}{l}\text { Type targeted } \\
\text { behavior }\end{array}$ & $\begin{array}{l}\text { Characteristics } \\
\text { providers }\end{array}$ & $\begin{array}{l}\text { Characteristics } \\
\text { patients; clinical } \\
\text { problem }\end{array}$ & Excluded patients & $\begin{array}{l}\text { Notes \& secondary } \\
\text { outcomes }^{\mathrm{a}}\end{array}$ \\
\hline Asplund & $\begin{array}{l}\text { Provider orientated: } \\
\text { clinical } \\
\text { multidisciplinary } \\
\text { teams }\end{array}$ & Patient outcome & $\begin{array}{l}\text { Staff recruited from } \\
\text { geriatric, medical and } \\
\text { surgical departments. } \\
\text { Consultants from both } \\
\text { geriatric and medical } \\
\text { departments had joint } \\
\text { responsibility for } \\
\text { medical care on the } \\
\text { ward, with the internist } \\
\text { having main } \\
\text { responsibility for acute } \\
\text { diagnosis and } \\
\text { treatment. }\end{array}$ & $\begin{array}{l}\text { All patients } \geq 70 \text { years } \\
\text { admitted acutely }\end{array}$ & $\begin{array}{l}\text { Requirement of } \\
\text { treatment in } \\
\text { specialized units } \\
\text { (e.g. IC, coronary } \\
\text { care, acute stroke } \\
\text { unit, renal unit) }\end{array}$ & $\begin{array}{l}\text { Ward organized } \\
\text { solely for the } \\
\text { purpose of this } \\
\text { study. } \\
\text { - Discharge } \\
\text { destination: ns } \\
\text { - LOS: + }\end{array}$ \\
\hline Counsell & $\begin{array}{l}\text { Provider orientated: } \\
\text { clinical } \\
\text { multidisciplinary } \\
\text { teams, patient- } \\
\text { centered care } \\
\text { Structural: changes in } \\
\text { physical structure, } \\
\text { facilities and } \\
\text { equipment }\end{array}$ & $\begin{array}{l}\text { Maintain or achieve } \\
\text { independence }\end{array}$ & $\begin{array}{l}\text { GCT, with nurse } \\
\text { having key role in } \\
\text { providing care. }\end{array}$ & $\begin{array}{l}\text { All patients } \geq 70 \text { years } \\
\text { admitted acutely }\end{array}$ & $\begin{array}{l}\text { Transferred from a } \\
\text { nursing facility or } \\
\text { another hospital } \\
\text { Requiring specialty } \\
\text { unit admission (e.g. } \\
\text { IC, coronary care, } \\
\text { telemetry, oncology) } \\
\text { Elective admissions } \\
\text { LOS <2 days }\end{array}$ & $\begin{array}{l}\text { - discharge des: NS } \\
\text { - mortality: NS } \\
\text { - LOS: NS } \\
\text { - costs: NS } \\
\text { - readmission: NS } \\
\text { - resource use: NS } \\
\text { - process variables: } \\
\text { nursing care plans, } \\
\text { discharge planning, } \\
\text { social work, physical } \\
\text { therapy, physical } \\
\text { restraint, high risk } \\
\text { medication: +; } \\
\text { urinary catheter, bed } \\
\text { rest: NS } \\
\text { - satisfaction } \\
\text { patients and } \\
\text { providers: + }\end{array}$ \\
\hline
\end{tabular}




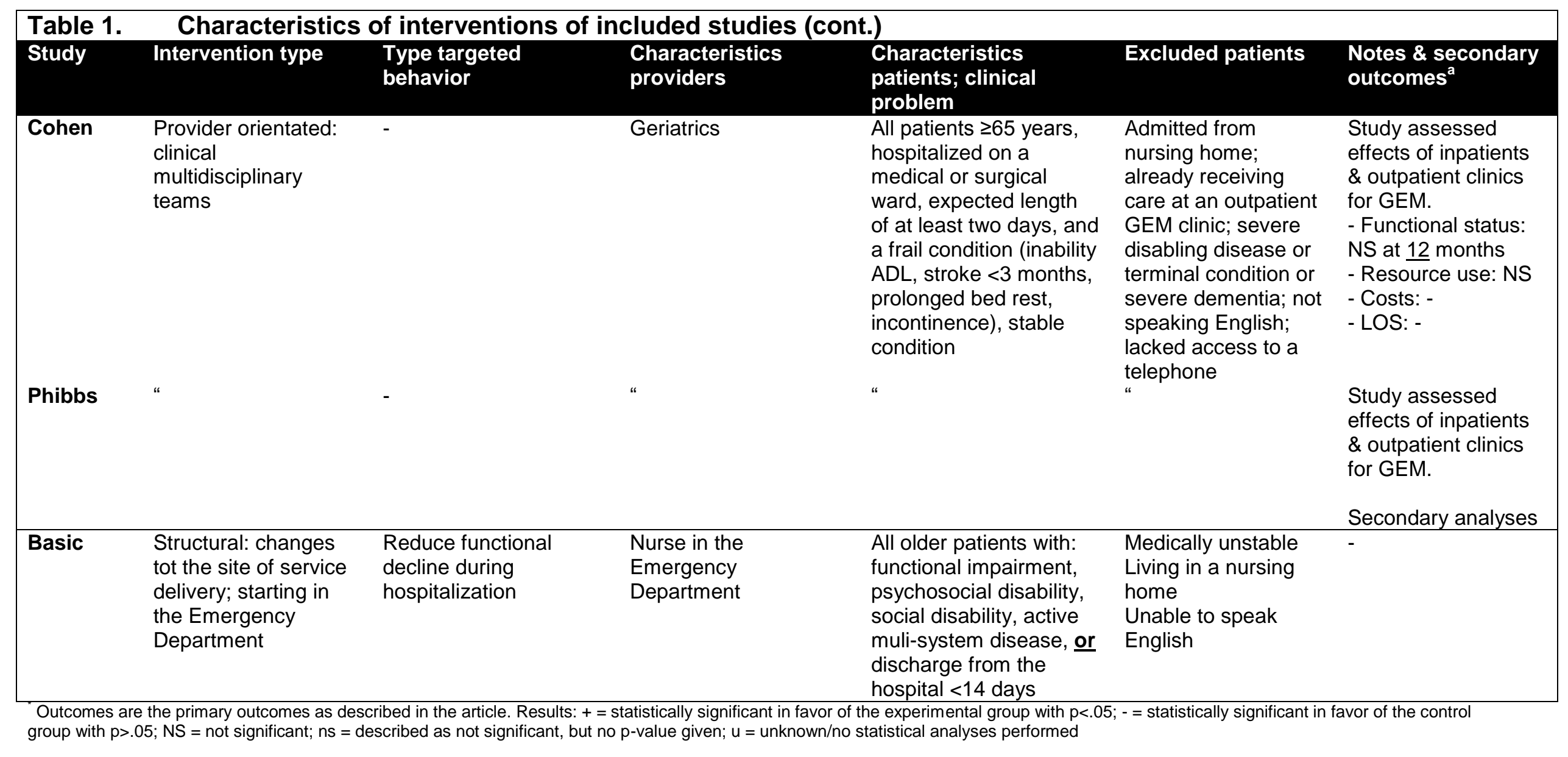




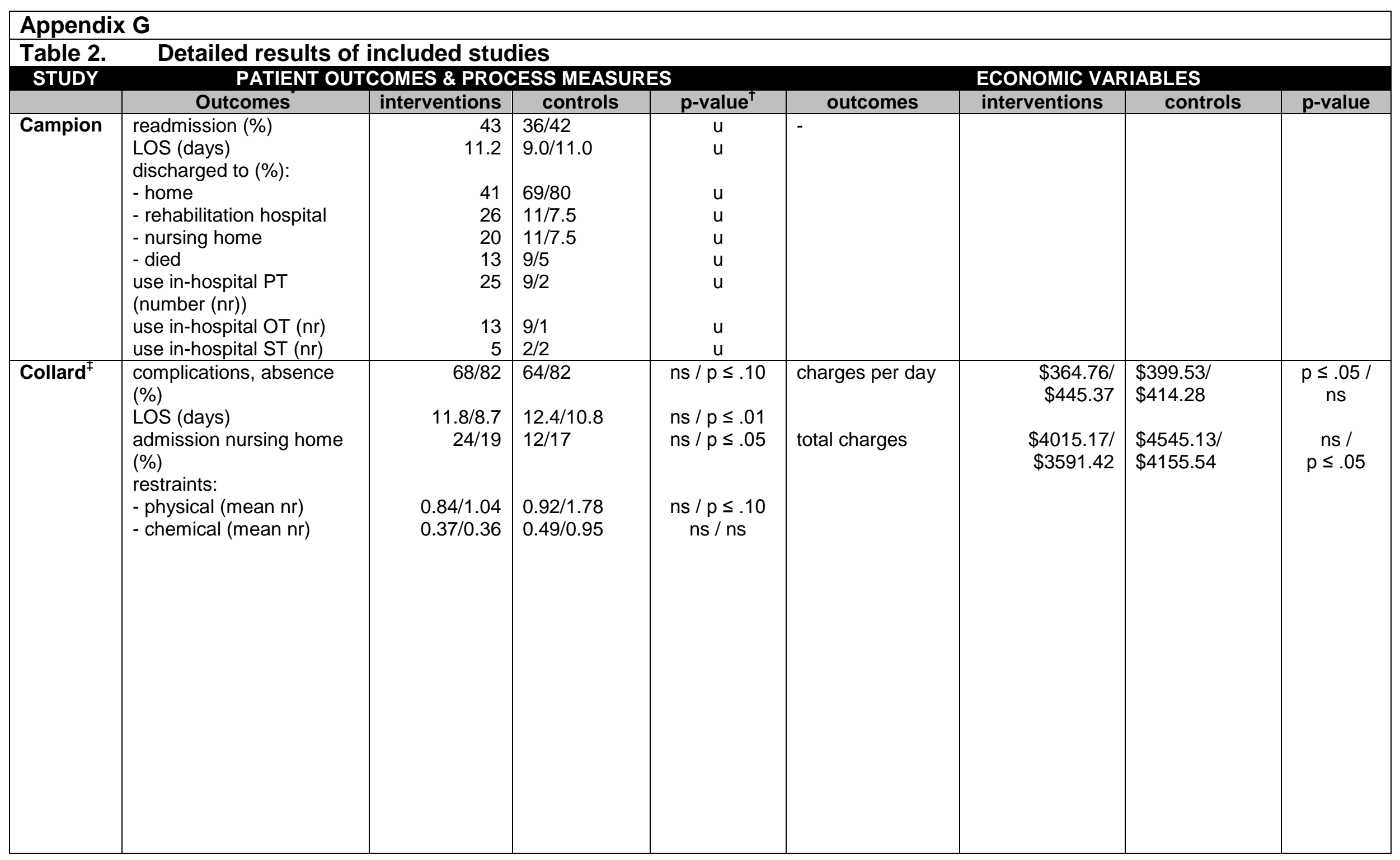




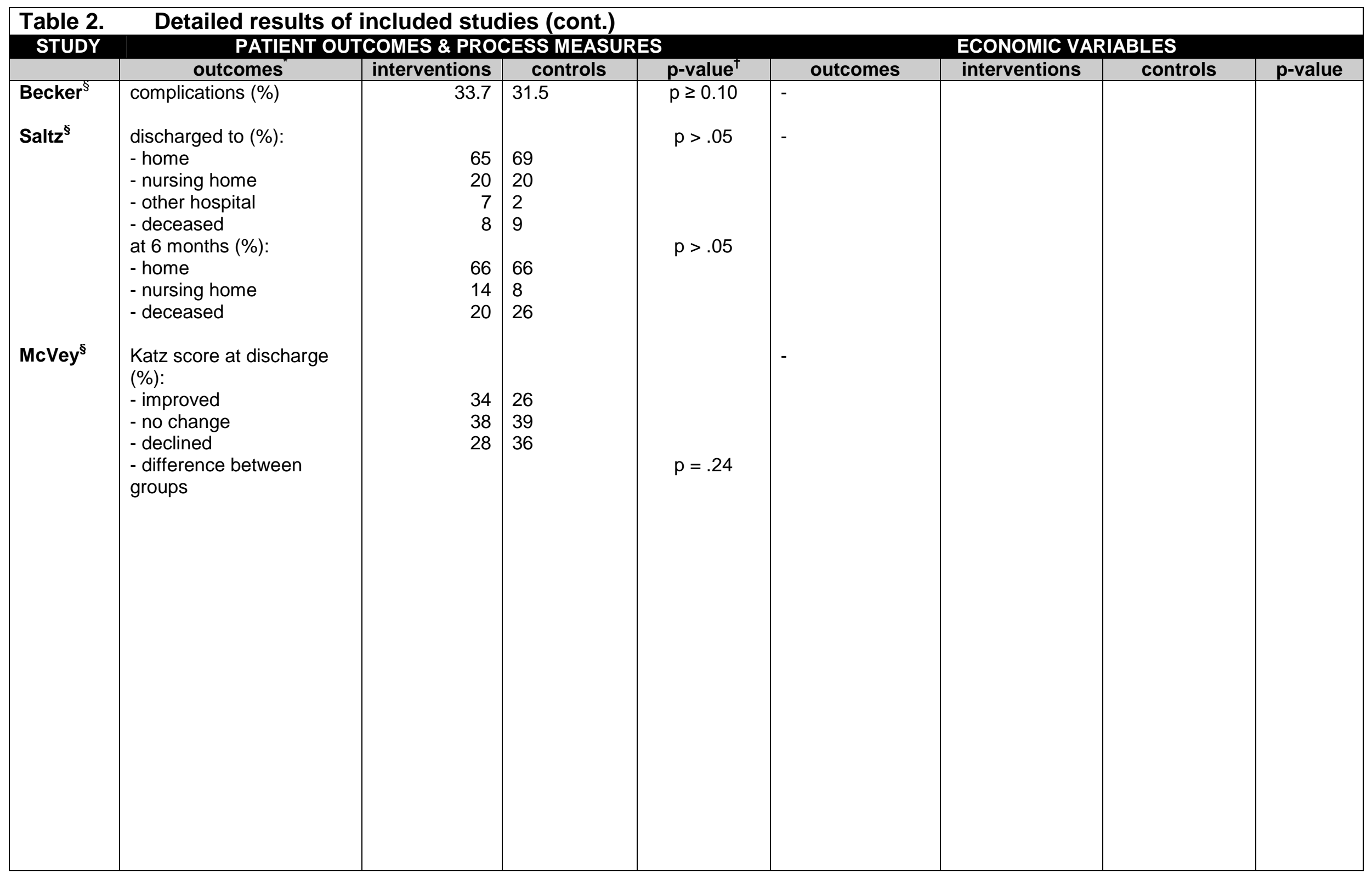




\begin{tabular}{|c|c|c|c|c|c|c|c|c|}
\hline \multicolumn{9}{|c|}{ Table 2. $\quad$ Detailed results of included studies (cont.) } \\
\hline Gayton & $\begin{array}{l}\text { Barthel index (score 0- } \\
100,6 \mathrm{~m}) \\
\text { PSPMSQ }(6 \mathrm{~m}) \\
\text { LOS (days) } \\
\text { discharged to (\%): } \\
\text { - community } \\
\text { - convalescence } \\
\text { - long-term care } \\
\text { - died } \\
\text { resource use post- } \\
\text { discharge }\end{array}$ & $\begin{array}{r}83.1 \pm 26.0 \\
2.9 \pm 3.2 \\
20.6 \pm 23.4 \\
\\
64.9 \\
8.6 \\
9.5 \\
14.9 \\
u\end{array}$ & $\begin{array}{l}81.7 \pm 28.5 \\
2.3 \pm 2.9 \\
20.6 \pm 25.3 \\
\\
58.8 \\
6.6 \\
12 \\
19.1 \\
u\end{array}$ & $\begin{array}{l}u \\
u \\
u \\
u \\
u \\
u \\
u \\
u\end{array}$ & 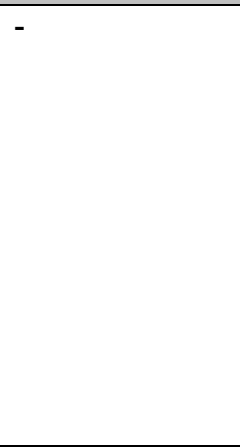 & & & \\
\hline $\begin{array}{l}\text { Hogan } \\
(1987)\end{array}$ & $\begin{array}{l}\text { Barthel index score } \\
\text { (change) } \\
\text { mental status scorell } \\
\text { (change) } \\
\text { LOS (days) } \\
\text { discharged nursing home } \\
\text { (\%) } \\
\text { referred in-hospital SW } \\
(\%) \\
\text { referred in-hospital PT } \\
(\%) \\
\text { referred in-hospital OT } \\
(\%) \\
\text { referred in-hospital ST } \\
(\%) \\
\text { referred in-hospital DT } \\
(\%) \\
\text { referrals community } \\
\text { services } \\
\text { prescribed oral } \\
\text { medications: } \\
\text { - change number } \\
\text { - percentage decrease }\end{array}$ & $\begin{array}{r}27.5 \pm 23.3 \\
1.5 \pm 1.4 \\
15.8 \pm 12.7 \\
3 \\
53 \\
44 \\
18 \\
0 \\
32 \\
1.3 \pm 0.6 \\
\\
0.04 \pm 0.27 \\
47\end{array}$ & $\begin{array}{l}19.8 \pm 19.4 \\
0.8 \pm 2.1 \\
14.2 \pm 13.3 \\
10 \\
43 \\
21 \\
0 \\
2 \\
21 \\
0.9 \pm 0.6\end{array}$ & $\begin{array}{c}n s \\
p \leq .01 \\
n s \\
n s \\
n s \\
p<.025 \\
p<.005 \\
n s \\
n s \\
p<.005\end{array}$ & - & & & \\
\hline
\end{tabular}




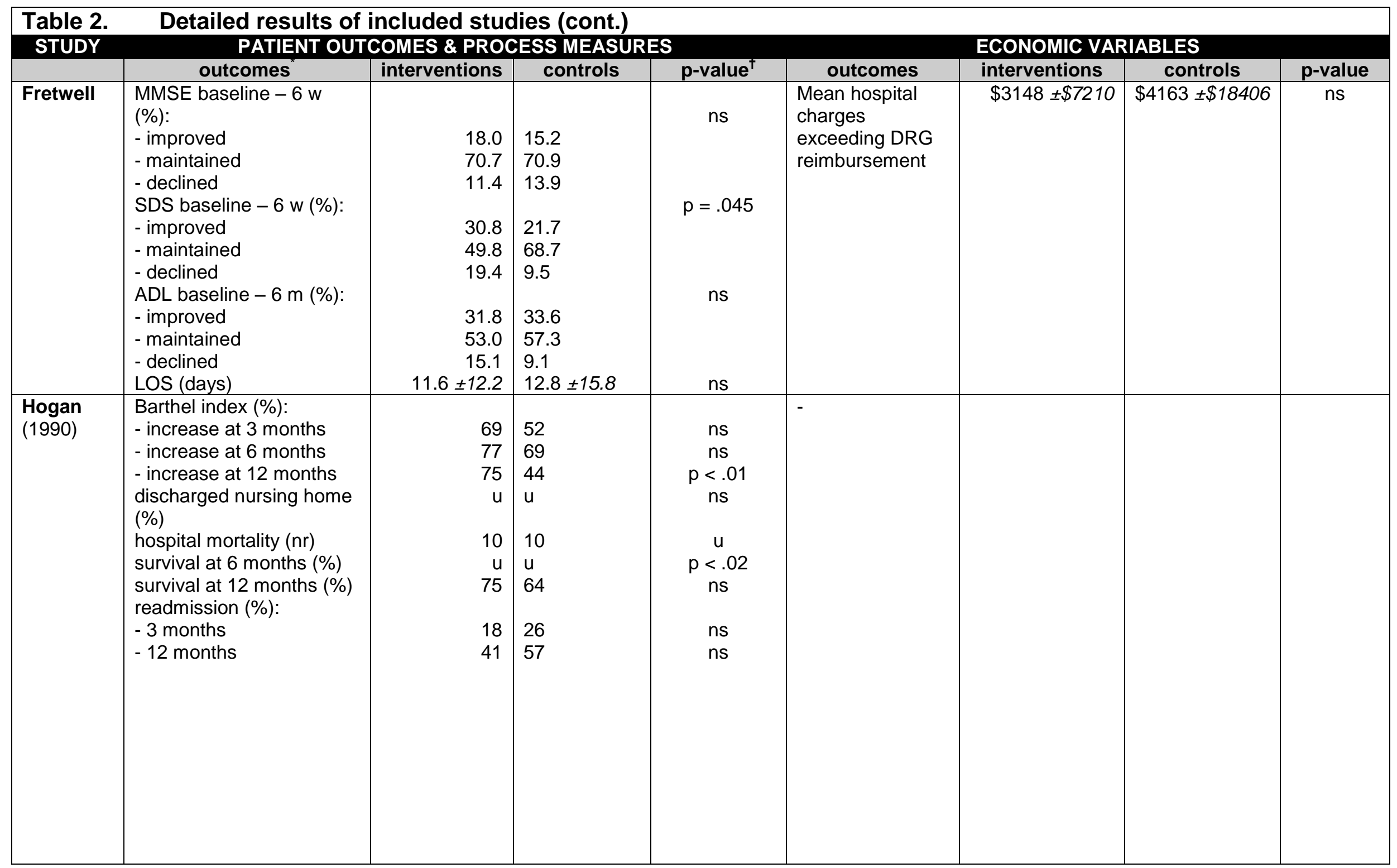




\begin{tabular}{|c|c|c|c|c|c|c|c|c|}
\hline \multirow{2}{*}{ STUDY } & \multicolumn{4}{|c|}{ PATIENT OUTCOMES \& PROCESS MEASURES } & \multicolumn{4}{|c|}{ ECONOMIC VARIABLES } \\
\hline & outcomes & interventions & controls & $p$-value ${ }^{\dagger}$ & outcomes & interventions & controls & p-value \\
\hline Inouye & $\begin{array}{l}\text { overall functional decline } \\
\text { between baseline and } \\
\text { discharge (\%) }\end{array}$ & $\begin{array}{r}42(95 \% \text { Cl, } \\
27-57) / \\
29(95 \% \text { Cl, } \\
15-42)\end{array}$ & $\begin{array}{l}34 \\
(95 \% \mathrm{Cl}, 25- \\
42)\end{array}$ & $\mathrm{u}$ & & & & \\
\hline Thomas & $\begin{array}{l}\text { Katz score (change \%): } \\
\text { - same } \\
\text { - worse } \\
\text { - better } \\
\text { mortality at } 6 \text { months (\%) } \\
\text { mortality at } 12 \text { month }(\%) \\
\text { readmissions (pp, } 6 \mathrm{~m}) \\
\text { LOS (days) } \\
\text { discharge destination } \\
\text { referrals community } \\
\text { services } \\
\text { post-discharge outpatient } \\
\text { physician office visits (nr } \\
\text { per patient) }\end{array}$ & $\begin{array}{r}61 \\
17 \\
22 \\
6 \\
10 \\
0.3 \\
9 \\
u \\
0.6 \\
\\
3.5\end{array}$ & $\begin{array}{l}70 \\
23 \\
7 \\
21 \\
20 \\
0.6 \\
10.1 \\
u \\
0.4 \\
4.6\end{array}$ & $\begin{array}{c}p=.17 \\
p=.01 \\
p=.08 \\
p=.02 \\
p=.20 \\
n s \\
p=.10 \\
p=.09\end{array}$ & & & & \\
\hline Winograd & $\begin{array}{l}\text { IADL score at } 12 \mathrm{~m}^{\|} \\
\text {MMSE score at } 12 \mathrm{~m}^{\pi} \\
\text { PSMS score at } 12 \mathrm{~m} \\
\text { morale at } 12 \mathrm{~m}^{\pi} \\
\text { survival at } 12 \mathrm{~m}(\%) \\
\text { readmissions (nr at } 12 \mathrm{~m} \text { ) } \\
\text { LOS (days) } \\
\text { discharged to }(\%) \text { : } \\
\text { - community } \\
\text { - sheltered living } \\
\text { - nursing home } \\
\text { - in-hospital mortality } \\
\text { hospital days (nr at } 12 \mathrm{~m} \text { ) } \\
\text { nursing home days } \\
\text { (nr } 12 \mathrm{~m} \text { ) } \\
\text { level of care at discharge } \\
\text { level of care at } 12 \mathrm{~m}\end{array}$ & $\begin{array}{r}4.6 \pm 2.8 \\
24.3 \pm 7.1 \\
3.6 \pm 2.0 \\
14.1 \pm 2.8 \\
59 \\
1.0 \pm 1.3 \\
24.8 \pm 22.0 \\
60 \\
9 \\
16 \\
14 \\
15 \pm 27 \\
35.9 \pm 74.5 \\
\\
1.1 \pm 0.4 \\
1.4 \pm 0.8\end{array}$ & $\begin{array}{l}5.2 \pm 3.1 \\
21.4 \pm 9.2 \\
4.0 \pm 2.1 \\
14.2 \pm 2.7 \\
64 \\
1.2 \pm 1.7 \\
26.7 \pm 33.0 \\
66 \\
10 \\
18 \\
6 \\
20 \pm 39 \\
25.8 \pm 63.1 \\
1.1 \pm 0.3 \\
1.4 \pm 0.7\end{array}$ & $\begin{array}{l}p=.69 \\
p=.02 \\
p=.91 \\
p=.23 \\
p=.43 \\
p=.46 \\
p=.91 \\
p=.34 \\
\\
p=.44 \\
p=.33 \\
p=.34 \\
p=.35\end{array}$ & & & & \\
\hline
\end{tabular}




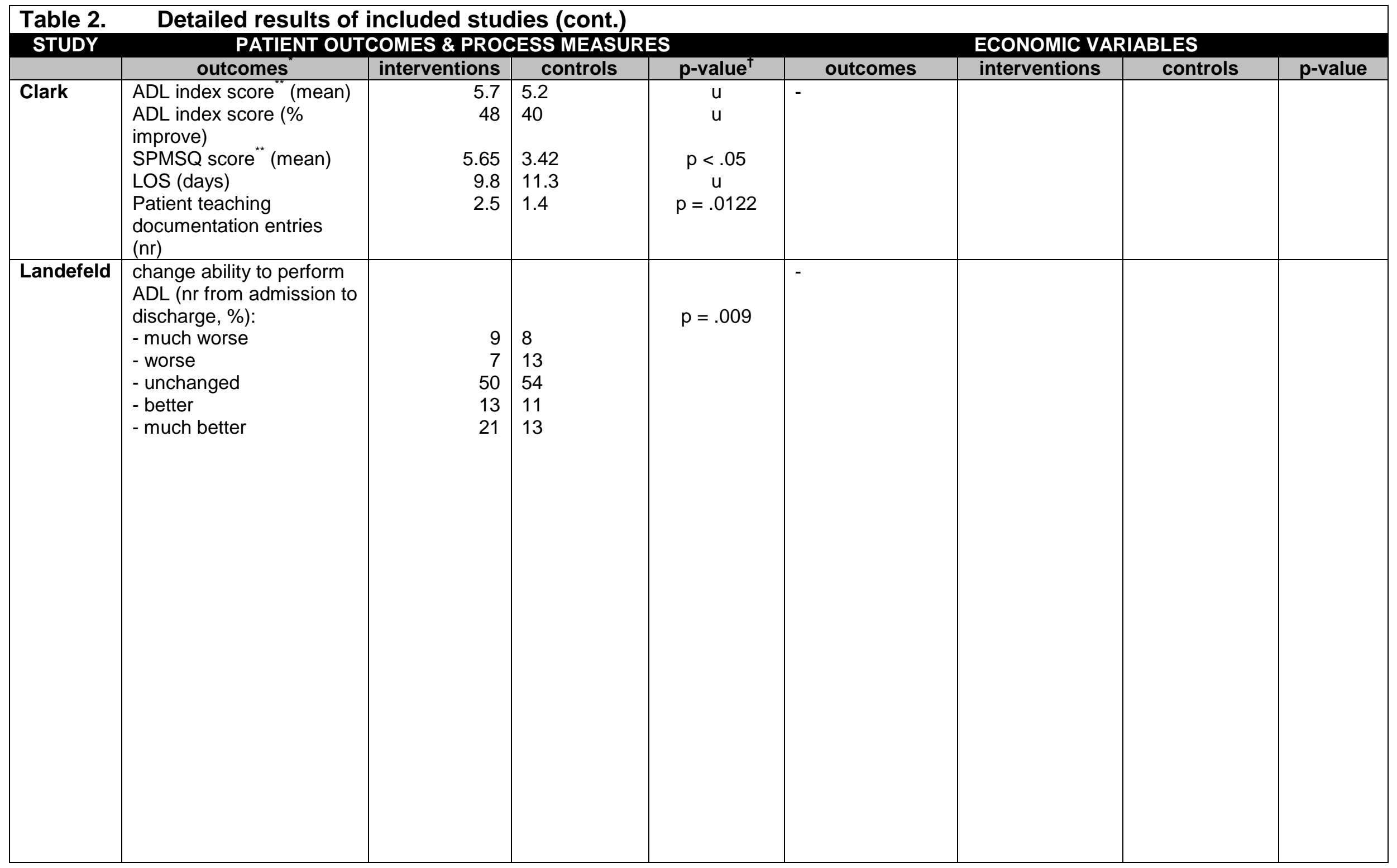




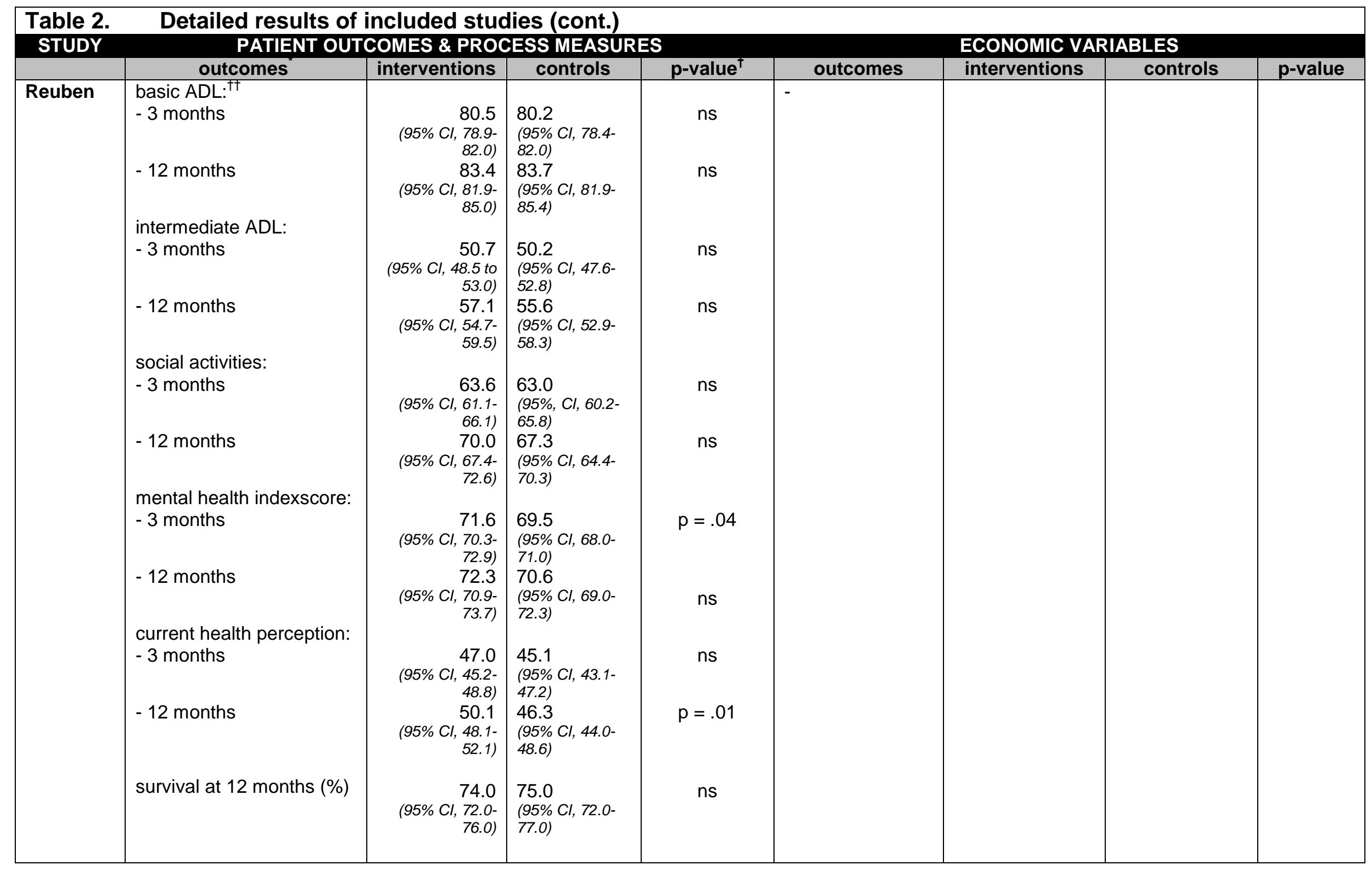




\begin{tabular}{|c|c|c|c|c|c|c|c|c|}
\hline \multirow[t]{2}{*}{ STUDY } & \multicolumn{4}{|c|}{ PATIENT OUTCOMES \& PROCESS MEASURES } & \multicolumn{4}{|c|}{ ECONOMIC VARIABLES } \\
\hline & outcomes & interventions & controls & p-value ${ }^{\dagger}$ & outcomes & interventions & controls & p-value \\
\hline Asplund & $\begin{array}{l}\text { poor global outcome (3 m, } \\
\%) \\
\text { readmissions ( } 3 \mathrm{~m}, \%) \\
\text { outpatient medical care } \\
\text { ( } 3 \mathrm{~m}, \text { nr visits): } \\
\text { - physician } \\
\text { - nurse } \\
\text { - PT/OT }\end{array}$ & \begin{tabular}{|r|} 
\\
\\
\\
\\
\\
\\
\\
\\
2.1 \\
$(95 \% \mathrm{Cl}, 1.8-$ \\
$2.4)$ \\
2.6 \\
$(95 \% \mathrm{Cl}, 1.9-$ \\
$3.6)$ \\
0.9 \\
$(95 \% \mathrm{Cl}, 0.3-$ \\
$1.5)$
\end{tabular} & $\begin{array}{l}34 \\
28 \\
\\
\\
\\
2.0 \\
(95 \% \mathrm{Cl}, 1.7- \\
2.2) \\
3.4 \\
(95 \% \mathrm{Cl}, 1.8- \\
5.0 \\
0.2 \\
(95 \% \mathrm{Cl}, 0.1- \\
0.3)\end{array}$ & $\begin{array}{c}\mathrm{ns} \\
R R 1.06(95 \% \\
C l, 0.84-1.34) \\
\mathrm{ns} \\
R R \quad 1.14(95 \% \\
C l, 0.92-1.43) \\
\mathrm{ns} \\
\mathrm{ns} \\
\mathrm{p}=.02\end{array}$ & $\begin{array}{l}\text { total costs ( } 3 \mathrm{~m} \text {, } \\
\text { SEK) }\end{array}$ & $\begin{array}{r}3600 \\
(95 \% \text { Cl, } 1200- \\
15200)\end{array}$ & $\begin{array}{l}3600 \\
(95 \% \text { Cl, 1200- } \\
14600)\end{array}$ & ns \\
\hline Counsell & $\begin{array}{l}\text { change in number of } \\
\text { independent ADL two } \\
\text { weeks before admission } \\
\text { to discharge (\%): } \\
\text { - improved } \\
\text { - maintained } \\
\text { - declined }\end{array}$ & $\begin{array}{r}9 \\
61 \\
30\end{array}$ & $\begin{array}{l}10 \\
56 \\
34\end{array}$ & $p=.33$ & - & & & \\
\hline
\end{tabular}




\begin{tabular}{|c|c|c|c|c|c|c|c|c|}
\hline \multirow[t]{2}{*}{ STUDY } & \multicolumn{4}{|c|}{ PATIENT OUTCOMES \& PROCESS MEASURES } & \multicolumn{4}{|c|}{ ECONOMIC VARIABLES } \\
\hline & outcomes & interventions & controls & p-value ${ }^{\dagger}$ & outcomes & interventions & controls & p-value \\
\hline Cohen $^{\mp \mp}$ & $\begin{array}{l}\text { HRQoL at } 12 \text { months: } \\
\text { - physical functioning } \\
\text { - physical limitations } \\
\text { - emotional limitations } \\
\text { - bodily pain } \\
\text { - energy } \\
\text { - mental health } \\
\text { - social activity } \\
\text { - general health } \\
\text { mortality at } 12 \text { months } \\
(\%)\end{array}$ & $\begin{array}{r}6.7 \\
34.0 \\
22.0 \\
24.9 \\
4.5 \\
4.5 \\
18.3 \\
-5.5 \\
21\end{array}$ & $\begin{array}{l}4.5 \\
29.8 \\
20.3 \\
20.0 \\
1.8 \\
2.5 \\
16.4 \\
-7.1 \\
21\end{array}$ & $\begin{array}{c}p=.30 \\
p=.13 \\
p=.58 \\
p=.01 \\
p=.12 \\
p=.24 \\
p=.48 \\
p=.32 \\
n s \\
\text { (OR } 0.95,95 \% \\
\text { Cl } 0.6-131)\end{array}$ & 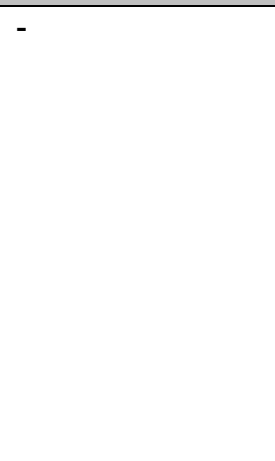 & & 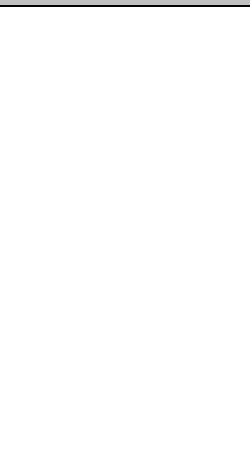 & \\
\hline Phibbs $^{\ddagger \ddagger}$ & $\begin{array}{l}\text { admission nursing home } \\
\text { (nr) } \\
\text { nursing home days (nr) }\end{array}$ & $\begin{array}{r}127 \\
21.2 \pm 2.4\end{array}$ & $\begin{array}{l}177 \\
28.4 \pm 2.7\end{array}$ & $\begin{array}{l}p=.001 \\
p=.003\end{array}$ & $\begin{array}{l}\text { nursing home } \\
\text { cost } \\
\text { total costs: } \\
\text { - index hospital } \\
\text { - after discharge }\end{array}$ & $\begin{array}{r}\$ 5853( \pm \$ 665) \\
\\
\$ 13449 \\
( \pm \$ 621) \\
\$ 22816 \\
( \pm \$ 1080)\end{array}$ & $\begin{array}{l}\$ 7828( \pm \$ 741) \\
\$ 10758 \\
( \pm \$ 592) \\
\$ 26533 \\
( \pm \$ 1201)\end{array}$ & $\begin{array}{l}p=.002 \\
p=.0001 \\
p=.03\end{array}$ \\
\hline Basic & $\begin{array}{l}\text { functional decline during } \\
\text { hospitalization } \\
\text { admission to the hospital } \\
\text { LOS }\end{array}$ & & & $\begin{array}{l}\text { OR } 1.26 \\
(95 \% \mathrm{Cl}, \\
0.48-3.30) \\
\\
\text { OR } 0.65 \\
(95 \% \mathrm{Cl} \\
0.25-1.70) \\
\text { HR } 1.06 \\
(95 \% \mathrm{Cl} \\
0.74-1.52)\end{array}$ & - & & & \\
\hline
\end{tabular}


Ssame study

status score was measured by mental status questionnaire, but was no further specified in article.

IADL: possible scores 0 through 8; lower score, better function.

MMSE: possible scores 0 through 30; higher score, better function.

Philidelphia Geriatric Center Morale Scale was used to measure morale, possible scores 0 through 18; lower score, better function.

PSMS: possible scores 0 through 18; lower score, better function.

"The index of Activities of Daily Living was used to assess functional status, which resulted in an overall grade. According to de Index, performance was summarized as scores 1 (independent) through 7 (dependent).

SPSMSQ: score $>3=$ some impairment, score $>8$ = severely impaired.

${ }^{t t}$ The questionnaire on functional and health status consisted of three scales from the Functional Status Questionnaire concerning basic ADL, intermediate ADL and social activities; the mental health index and current-health-perceptions scale from the Medical Outcomes Study; and items from the Katz index of ADL, modified. All scores were standardized in a rang of 0 to 100 , with 100

indicating best function.
${ }_{\sharp}$ same study (secondary analyses Phibbs)

${ }^{8 s}$ HRQoL was assessed on the basis of the Medical Outcomes Study 36-Item Short-Form General Health Survey (SF-36), scale 0 through 100 


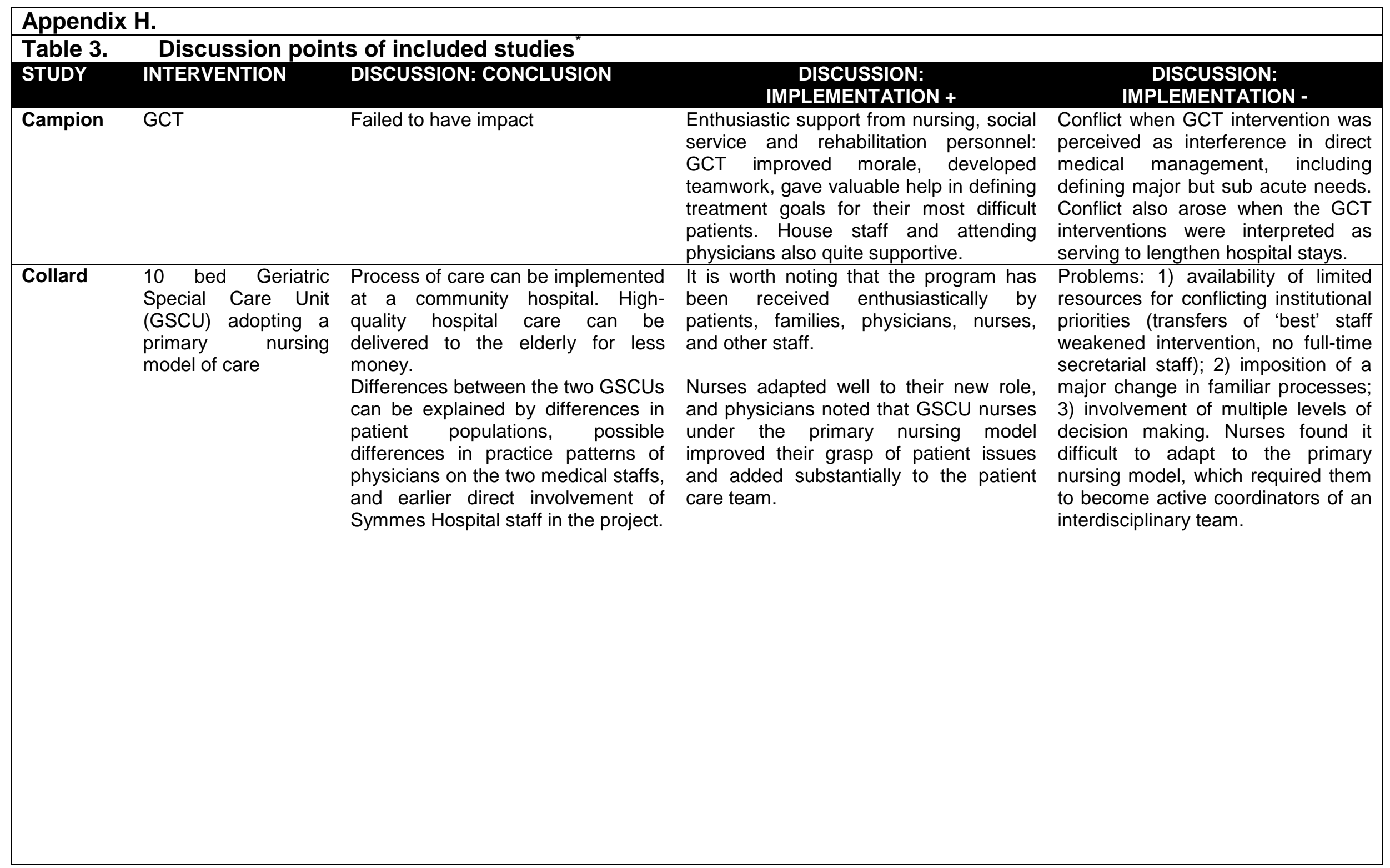




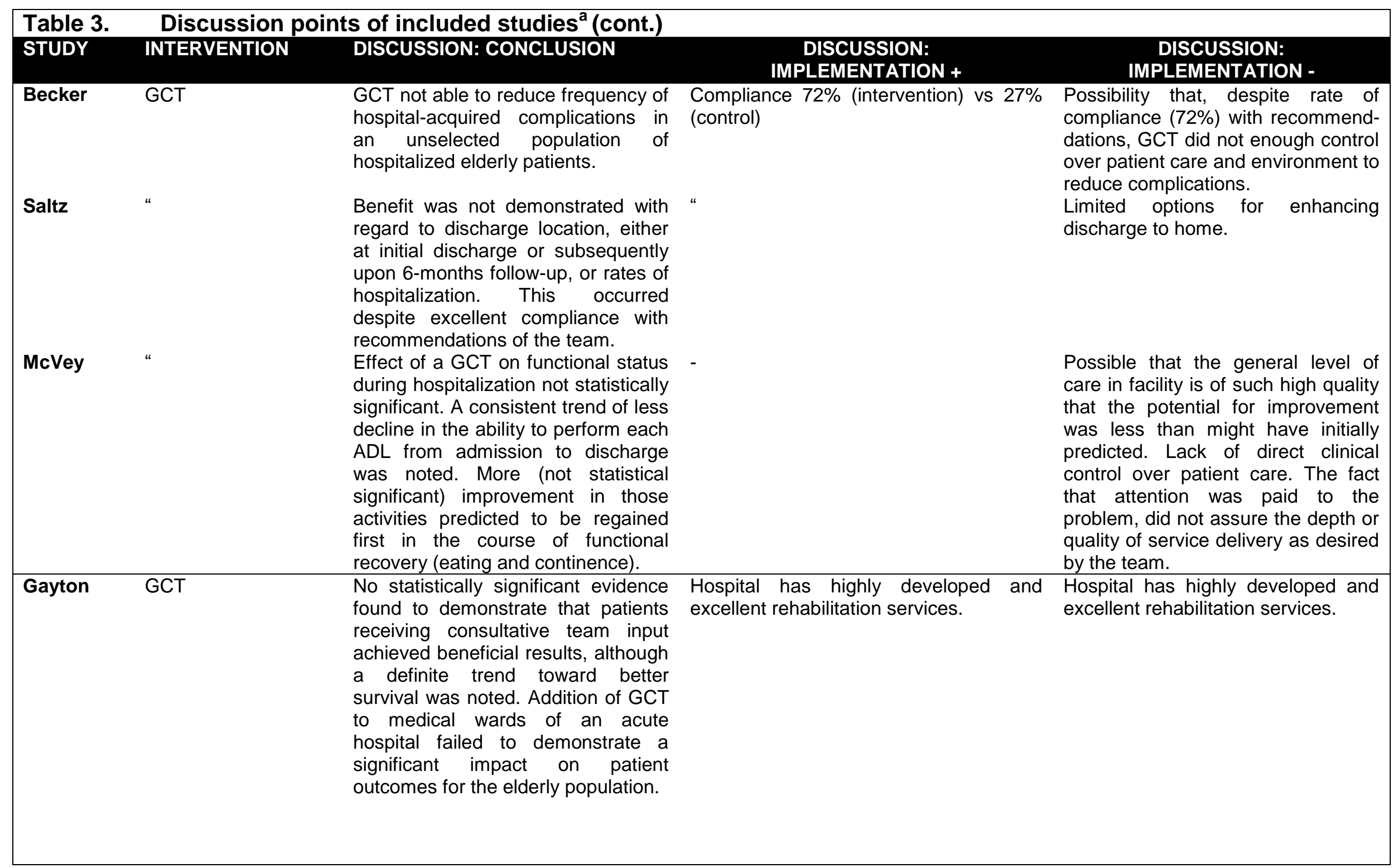




\begin{tabular}{|c|c|c|c|c|}
\hline $\begin{array}{l}\text { Table } 3 . \\
\text { STUDY }\end{array}$ & $\begin{array}{l}\text { Discussion poin } \\
\text { INTERVENTION }\end{array}$ & $\begin{array}{l}\text { s of included studies }{ }^{\text {a }} \text { (cont.) } \\
\text { DISCUSSION: CONCLUSION }\end{array}$ & $\begin{array}{c}\text { DISCUSSION: } \\
\text { IMPLEMENTATION + }\end{array}$ & $\begin{array}{l}\text { DISCUSSION: } \\
\text { IMPLEMENTATION - }\end{array}$ \\
\hline $\begin{array}{l}\text { Hogan } \\
(1987)\end{array}$ & GCT & $\begin{array}{l}\text { Appear to show benefit to health of } \\
\text { the intervention group and increased } \\
\text { use of health care resources. } \\
\text { Indication that functional disability is } \\
\text { common in elderly patients admitted } \\
\text { to hospital, geriatric consultation } \\
\text { service can have beneficial effect on } \\
\text { their management. }\end{array}$ & $\begin{array}{l}\text { Situation lent itself to such a study. The } \\
\text { consultation service was the first formal } \\
\text { geriatric program within the institution. } \\
\text { Study the effect of an isolated program } \\
\text { in new territory. } \\
\text { Goodwill of attending staff was } \\
\text { abundant. }\end{array}$ & nor. \\
\hline Fretwell & $\begin{array}{lr}\text { Senior Care } & \text { Unit, } 18 \\
\text { beds, } & \text { geriatric } \\
\text { assessment } & \text { team } \\
\text { (GAT) } & \end{array}$ & $\begin{array}{l}\text { Findings confirm that a GCT can be } \\
\text { instituted within a nursing unit of a } \\
\text { community hospital without } \\
\text { increasing LOS or hospital charges. } \\
\text { Found no significant differences in } \\
\text { discharge destination, or functional } \\
\text { and mental status. Only sign. effect } \\
\text { was higher rate of improvement in } \\
\text { mood of patients who were } \\
\text { depressed at admission. }\end{array}$ & Using existing hospital personnel. & $\begin{array}{l}\text { Possible failure of the attending } \\
\text { physician to implement the } \\
\text { recommendations of the GCT. } \\
\text { Contamination. Inclusion of } \\
\text { individuals who might not have been } \\
\text { able to respond to the interventions. } \\
\text { Insufficient intervention. Insensitivity } \\
\text { of outcome measures. Lack of } \\
\text { control over post-hospital care. }\end{array}$ \\
\hline $\begin{array}{l}\text { Hogan } \\
(1990)\end{array}$ & GCT & $\begin{array}{l}\text { No statistically significant differences } \\
\text { at time of discharge. Follow-up } \\
\text { showed beneficial effects extending } \\
\text { up to a year. GCT patients showed } \\
\text { improved survival, improved } \\
\text { functional capabilities and a trend } \\
\text { towards decreased reliance on } \\
\text { hospital and nursing homes. GCT } \\
\text { programs are effective. }\end{array}$ & $\begin{array}{l}\text { Consideration that the main beneficial } \\
\text { effects arise from the follow-up care } \\
\text { provided to patients, in an area where } \\
\text { the GCTs' familiarity with local } \\
\text { community resources and how to } \\
\text { mobilize them would lead to specific } \\
\text { benefits for patients. }\end{array}$ & $\begin{array}{l}\text { Not all recommendations of the GCT } \\
\text { were acted upon. }\end{array}$ \\
\hline
\end{tabular}




\begin{tabular}{|c|c|c|c|c|}
\hline STUDY & INTERVENTION & DISCUSSION: CONCLUSION & $\begin{array}{c}\text { DISCUSSION: } \\
\text { IMPLEMENTATION + }\end{array}$ & $\begin{array}{c}\text { DISCUSSION: } \\
\text { IMPLEMENTATION - }\end{array}$ \\
\hline Inouye & $\begin{array}{l}\text { Yale Geriatric Care } \\
\text { Program, nursing- } \\
\text { centered model of } \\
\text { care }\end{array}$ & $\begin{array}{l}\text { Found no effectiveness in overall } \\
\text { analyses in preventing functional } \\
\text { decline. The Yale Geriatric Care } \\
\text { program is a new model of care that } \\
\text { can effectively decrease functional } \\
\text { decline in high-risk elderly } \\
\text { hospitalized medical patients. The } \\
\text { intervention appears to be feasible } \\
\text { to implement and would serve } \\
\text { elderly patients through-out the } \\
\text { hospital setting. }\end{array}$ & $\begin{array}{l}\text { Geriatric Resource Nurses were regular } \\
\text { staff nurses who underwent special } \\
\text { training in geriatric nursing. Intervention } \\
\text { staff were readily available and costs of } \\
\text { the intervention were minimized. }\end{array}$ & $\begin{array}{l}\text { Lack of bed availability on the } \\
\text { medical service, leading to intense } \\
\text { pressure on admissions, made it } \\
\text { impossible to randomize patients to } \\
\text { intervention and usual care units. }\end{array}$ \\
\hline Thomas & GCT & $\begin{array}{l}\text { Conclude that short-term mortality } \\
\text { can be reduced in a community } \\
\text { inpatient acute hospital setting by a } \\
\text { GCT. Important differences in } \\
\text { mortality remain after } 1 \text { year of } \\
\text { follow-up. Trends towards improved } \\
\text { functional status and fewer hospital } \\
\text { readmissions favor the intervention } \\
\text { group. }\end{array}$ & $\begin{array}{l}\text { Team had recently been introduced. } \\
\text { Recommendations from the GCT were } \\
\text { rarely ignored. The community setting } \\
\text { may have allowed for greater impact on } \\
\text { attending physicians. Hospital resources } \\
\text { to implement recommendations readily } \\
\text { available. Inpatient GCT's enjoy wide } \\
\text { distribution throughout the hospital with } \\
\text { potential to interact with all specialties, } \\
\text { team work is fostered. }\end{array}$ & - \\
\hline Winograd & GCT & $\begin{array}{l}\text { Found that the GCT did not have an } \\
\text { effect on improving discharge } \\
\text { disposition, functional status, level of } \\
\text { care in the year of follow-up, } \\
\text { utilization of hospitals, nursing } \\
\text { homes, or other healthcare services. } \\
\text { The single positive outcome was an } \\
\text { improvement in mental status. } \\
\text { Conclude that the trial was negative. } \\
\text { In conclusion, cannot say whether } \\
\text { GCT is effective or ineffective. }\end{array}$ & $\begin{array}{l}\text { Trial was performed shortly after the } \\
\text { service was created. }\end{array}$ & $\begin{array}{l}\text { Direct patient care was provided } \\
\text { only when regular ward staff were } \\
\text { unable to provide services because } \\
\text { of inadequate staffing, primarily } \\
\text { social services. Compliance was } \\
\text { poorest for recommendations that } \\
\text { required staff time, effort, or } \\
\text { understanding of geriatric } \\
\text { syndromes. Available resources } \\
\text { were often unpredictable. Services } \\
\text { ordered were often not provided. } \\
\text { Rehabilitations services were often } \\
\text { delayed for 5-7 days. }\end{array}$ \\
\hline
\end{tabular}




\begin{tabular}{|c|c|c|c|c|}
\hline \multicolumn{5}{|c|}{ Discussion points of included studies ${ }^{\mathrm{a}}$ (cont.) } \\
\hline STUDY & INTERVENTION & DISCUSSION: CONCLUSION & $\begin{array}{c}\text { DISCUSSION: } \\
\text { IMPLEMENTATION + }\end{array}$ & $\begin{array}{l}\text { DISCUSSION: } \\
\text { IMPLEMENTATION - }\end{array}$ \\
\hline Clark & Dayroom, nurses & $\begin{array}{l}\text { Results of the study indicate that } \\
\text { patients who are } 85+\text { years, } \\
\text { admitted with mental status change, } \\
\text { syncope not specifically cardiac, a } \\
\text { fall history or with sepsis or infection, } \\
\text { could benefit from care provided in } \\
\text { an environment designed to meet } \\
\text { their specific needs. Decreased } \\
\text { restraint \& sitter use, LOS and } \\
\text { adverse events in a group who were } \\
\text { more impaired at baseline. }\end{array}$ & $\begin{array}{l}\text { No increase in staffing for the program. } \\
\text { In-service training and geriatric rounds } \\
\text { were provided for unit nurses by the } \\
\text { geriatrician for } 6 \text { months prior to } \\
\text { implementing the dayroom program. } \\
\text { Patient outcomes and LOS improved } \\
\text { with only limited environmental and } \\
\text { staffing changes. }\end{array}$ & $\begin{array}{l}\text { Dayroom could only accommodate } 4 \\
\text { patients. }\end{array}$ \\
\hline Landefeld & $\begin{array}{l}\text { Acute Care for Elders } \\
\text { (ACE) unit }\end{array}$ & $\begin{array}{l}\text { This RCT provides evidence that } \\
\text { specific changes in the provision of } \\
\text { acute hospital care can improve the } \\
\text { ability of a heterogeneous group of } \\
\text { older patients hospitalized with } \\
\text { acute illnesses to perform ADL at } \\
\text { the time of discharge, and can } \\
\text { reduce the frequency of discharge to } \\
\text { institutions for long-term care. }\end{array}$ & $\begin{array}{l}\text { This intervention program may } \\
\text { complement disease-specific or } \\
\text { treatment-specific efforts to improve } \\
\text { patients' outcomes. }\end{array}$ & - \\
\hline
\end{tabular}




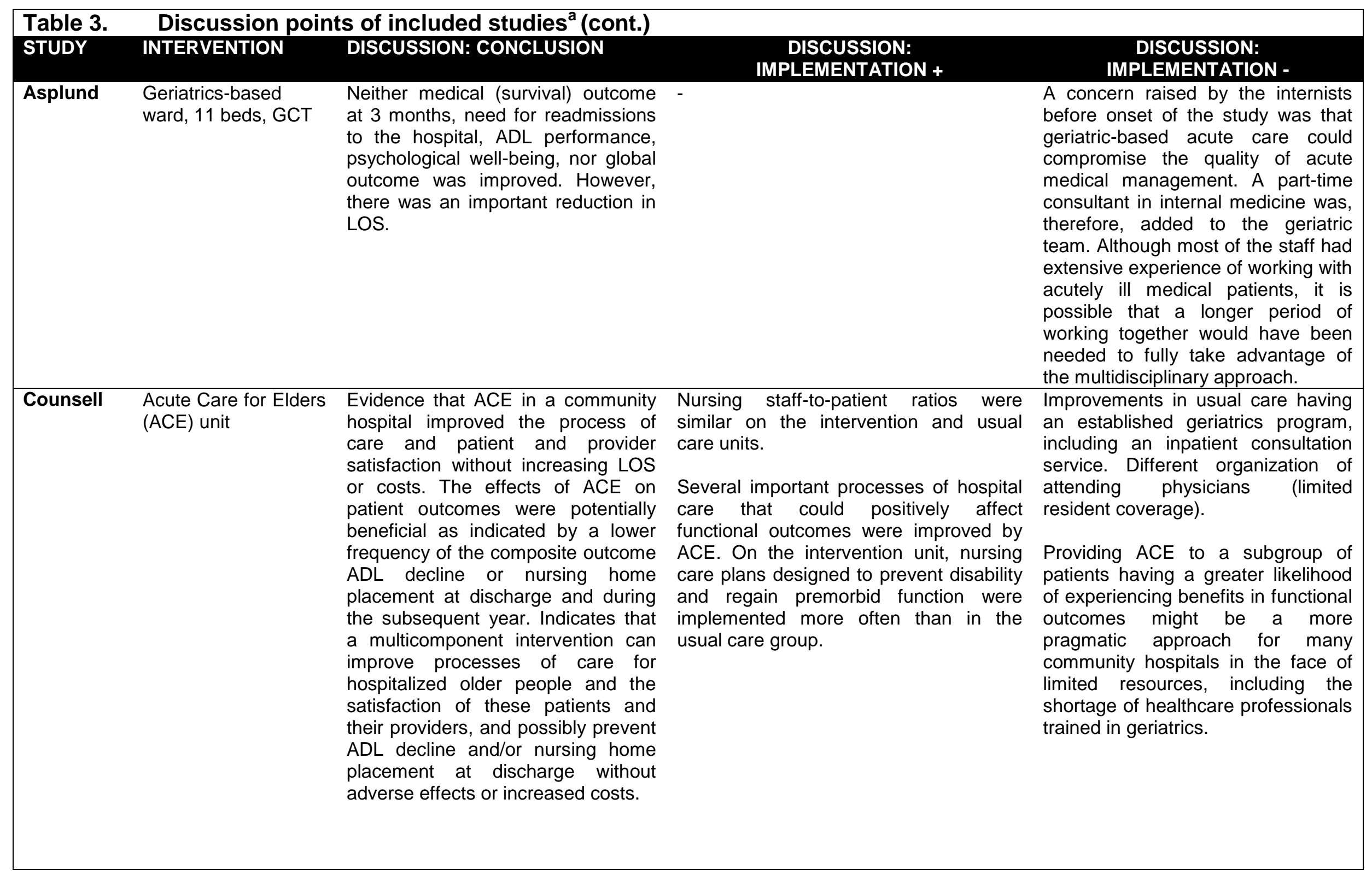




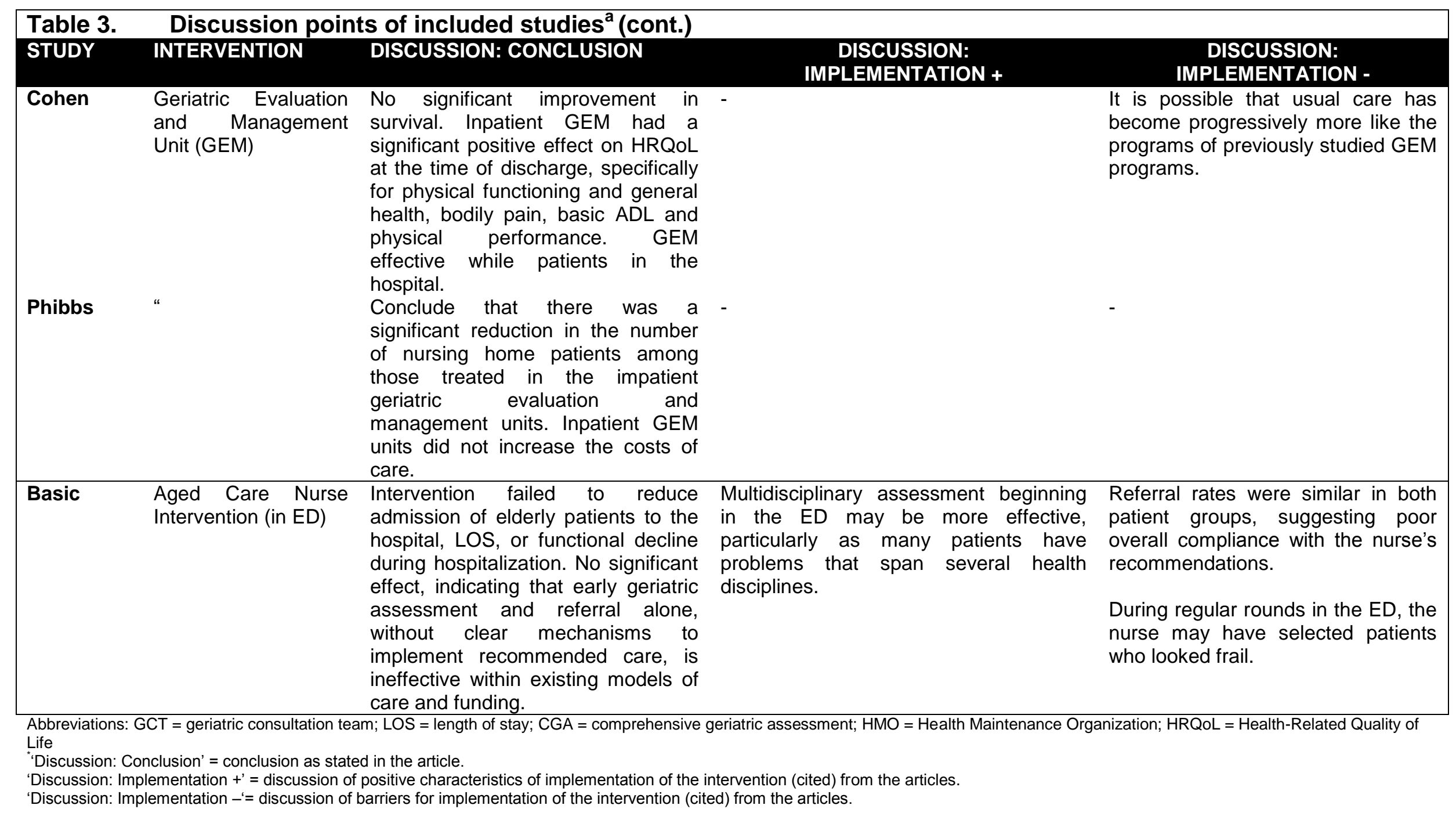




\section{Reference List}

(1) Hogan DB, Fox RA, Badley BW, Mann OE. Effect of a geriatric consultation service on management of patients in an acute care hospital. CMAJ 1987 April 1;136(7):713-7.

(2) Winograd CH, Gerety MB, Lai NA. A Negative Trial of Inpatient Geriatric Consultation: Lessons Learned and Recommendations for Future Research. Arch Intern Med 1993 September 13;153(17):2017-23.

(3) Reuben DB, Borok GM, Wolde-Tsadik G et al. A Randomized Trial of Comprehensive Geriatric Assessment in the Care of Hospitalized Patients. N Engl J Med 1995 May 18;332(20):1345-50.

(4) Fretwell MD, Raymond PM, McGarvey ST et al. The Senior Care Study. A Controlled Trial of a Consultative/Unit-Based Geriatric Assessment Program in Acute Care. J Am Geriatr Soc 1990 October;38(10):1073-81.

(5) Clark LR, Fraaza V, Schroeder S, Maddens ME. Alternative Nursing Environments: Do They Affect Hospital Outcomes? J Gerontol Nurs 1995 November;21(11):32-8.

(6) Landefeld CS, Palmer RM, Kresevic DM, Fortinsky RH, Kowal J. A Randomized Trial of Care in a Hospital Medical Unit Especially Designed to Improve the Functional Outcomes of Acutely Ill Older Patients. N Engl J Med 1995 May 18;332(20):1338-44.

(7) Hogan DB, Fox RA. A Prospective Controlled Trial of a Geriatric Consultation Team in an Acute-care Hospital. Age Ageing 1990 March;19(2):107-13.

(8) Cohen HJ, Feussner JR, Weinberger M et al. A Controlled Trial of Inpatient and Outpatient Geriatric Evaluation and Management. $N$ Engl J Med 2002 March 21;346(12):905-12.

(9) Thomas DR, Brahan R, Haywood BP. Inpatient Community-Based Geriatric Assessment Reduces Subsequent Mortality. J Am Geriatr Soc 1993 February;41(2):101-4.

(10) Collard AF, Bachman SS, Beatrice DF. Acute Care Delivery for the Geriatric Patient: An Innovative Approach. QRB Qual Rev Bull 1985 June;11(6):180-5.

(11) Phibbs CS, Holty JE, Goldstein MKM et al. The Effect of Geriatrics Evaluation and Management on Nursing Home Use and Health Care Costs: Results From a Randomized Trial. [Report]. Med Care 2006 January;44(1):91-5. 
(12) Asplund K, Gustafson Y, Jacobsson C et al. Geriatric-Based versus General Ward for Older Acute Medical Patients: a Randomized Comparison of Outcomes and Use of Resources. J Am Geriatr Soc 2000 November;48(11):1381-8.

(13) Campion EW, Jette A, Berkman B. An Interdisciplinary Geriatric Consultation Service: A Controlled Trial. J Am Geriatr Soc 1983 December;31(12):792-6.

(14) Becker PM, McVey LJ, Saltz CC, Feussner JR, Cohen HJ. Hospital-Acquired Complications in a Randomized Controlled Clinical Trial of a Geriatric Consultation Team. JAMA 1987 May 1;257(17):2313-7. 
Potentially relevant studies identified by database search and screened for relevance by title and available abstract $(\mathrm{n}=1175)$

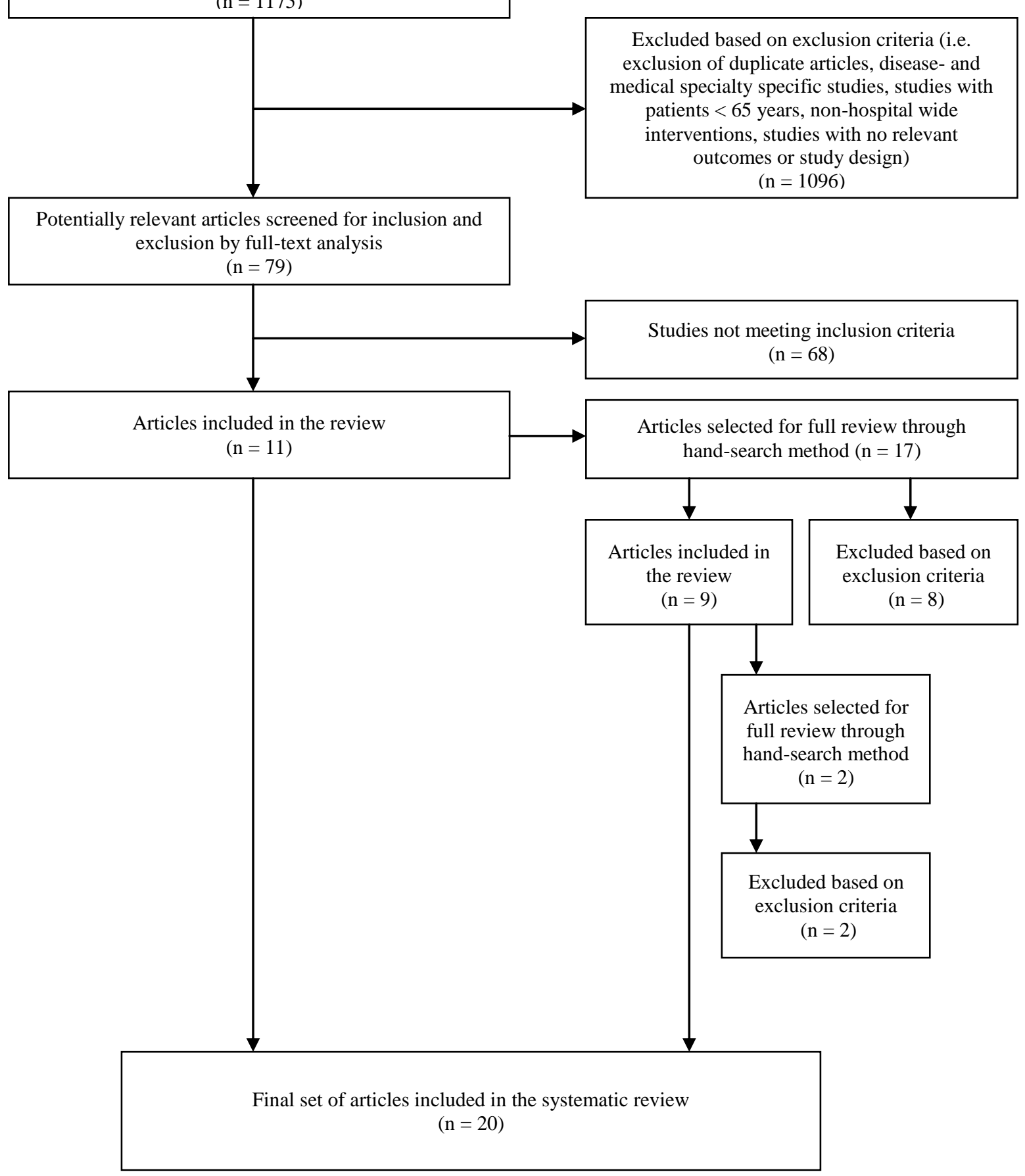

Figure 1. Flow-diagram of the selection of articles included in this systematic review. 